\title{
An Efficient Adaptive-Deniable-Concurrent Non-malleable Commitment Scheme
}

\author{
Seiko Arita \\ 2010.3.9. \\ Graduate School of Information Security, \\ Institute of Information Security, Japan
}

\begin{abstract}
It is known that composable secure commitments, that is, concurrent non-malleable commitments exist in the plain model, based only on standard assumptions such as the existence of claw-free permutations or even one-way functions. Since being based on the plain model, the deniability of them is trivially satisfied, and especially the latter scheme satisfies also adaptivity, hence it is adaptivedeniable-concurrent non-malleable. However, those schemes cannot be said to be practically efficient. We show a practically efficient (string) adaptive-deniable-concurrent commitment scheme is possible under a global setup model, called global CRS-KR model.
\end{abstract}

keywords: commitment schemes, adaptivity, deniability, concurrency, non-malleability.

\section{Introduction}

As advanced security properties, there are emerging concerns on composability, deniability and adaptivity of cryptographic protocols. The composability, culminating in Universal Composability (UC) [4], requires secure protocols remain secure even if they are composed with another protocols. The deniability requires secure protocols leave no evidence of their executions. The adaptivity requires secure protocols remain secure (to some reasonable extent) even if some honest parties are corrupted on way of their executions.

In this paper, we want to construct practically efficient commitment protocols with those advanced security properties in some global setup model.

It is known that composable secure commitments ${ }^{1}$, that is, concurrent non-malleable commitments exist in the plain model, based only on standard assumptions such as the existence of claw-free permutations $[22,20]$ or even one-way functions [18]. Since being based on the plain model, the deniability of them is trivially satisfied, and especially the scheme of [20] satisfies also adaptivity, hence it is already an adaptive-deniable-concurrent non-malleable commitment scheme. However, those schemes cannot be said to be practically efficient.

We show a practically efficient (string) adaptive-deniable-concurrent non-malleable commitment scheme is possible under a global setup model, called global CRS-KR model.

- We define a notion of adaptive-deniable-concurrent non-malleable commitments, that captures the three advanced properties all at once for commitment schemes in the global CRS-KR model.

- We define, as a more-easy-to-prove property, a straight-line equivocal-extractability of commitment schemes and prove that it (with some other auxiliary properties) yields the adaptivedeniable-concurrent non-malleability in the global CRS-KR model.

\footnotetext{
${ }^{1}$ From now on, the composability always addresses self-composition in this paper.
} 
- We construct a straight-line equivocal-extractable commitment scheme in the global CRS-KR model, under the decisional linear assumption and the knowledge of exponent assumption on bilinear groups. The scheme is efficient and practical, using a constant number of pairing computations and three-round exchanges of linear-size messages.

Related works. In the literature there exists only one (to our knowledge) practically-efficient commitment scheme by Canetti, Dodis, Pass and Walfish [5], that establishes the adaptive-deniableconcurrent non-malleability. Their scheme relies on a global setup model, called augmented CRS model (a kind of global CRS model with an augmented functionality) and is proved to be adaptively UC-secure in that model. It uses four-round message exchanges of square size $O\left(k^{2}\right)$ of security parameter $k$. We believe that our global CRS-KR model is comparable to the augmented CRS model and our scheme, using three-round exchanges of messages of linear size $O(k)$, is more efficient than their scheme.

\section{An Adaptive-Deniable-Concurrent Non-malleable Commitment}

\subsection{Commitment Schemes}

In this paper, we deal with commitments in a tag-based manner. On input value $v$ and tag $t$, sender $\mathcal{S}$ commits to $v$ through a transcript $c$ for receiver $\mathcal{R}$ using a CRS $\sigma$ (Commitment phase). Later, using local output $d$ of the commitment phase, $\mathcal{S}$ decommits $c$ to the value $v$ using the same tag $t$ and CRS $\sigma$ (Decommitment phase). Hiding property requires even adversarial receiver $\mathcal{R}^{*}$ cannot know the value $v$ under the commitment $c$ in the commitment phase. Binding property requires even adversarial sender $\mathcal{S}^{*}$ cannot decommit a same commitment $c$ to two different values. More precisely,

Definition 1 (Commitment Schemes). A triple $\Sigma=(\mathcal{K}, \mathcal{S}, \mathcal{R})$ of probabilistic polynomial time algorithms is called a (tag-based) commitment scheme if it satisfies the following three properties:

- (Correctness) For any value $v \in\{0,1\}^{k}$ and any tag, the probability

$$
\operatorname{Pr}\left[\sigma \leftarrow \mathcal{K}\left(1^{k}\right),(d, c) \leftarrow\left\langle\mathcal{S}_{\sigma, t a g}(v), \mathcal{R}_{\sigma, t a g}\right\rangle,\left(-, v^{\prime}\right) \leftarrow\left\langle\mathcal{S}_{\sigma, t a g}(d), \mathcal{R}_{\sigma, t a g}(c)\right\rangle: v=v^{\prime}\right]
$$

is negligibly close to 1 (with respect to $k$ ).

- (Hiding) For any adversary $\mathcal{R}^{*}$ and any tag, the probability

$$
\operatorname{Pr}\left[\sigma \leftarrow \mathcal{K}\left(1^{k}\right),\left(v_{0}, v_{1}, s\right) \leftarrow \mathcal{R}^{*}(\sigma, \text { tag }), b \stackrel{\$}{\leftarrow}\{0,1\},\left(-, b^{\prime}\right) \leftarrow\left\langle\mathcal{S}_{\sigma, t a g}\left(v_{b}\right), \mathcal{R}^{*}(s)\right\rangle: b=b^{\prime}\right]
$$

is negligibly close to $1 / 2$ (with respect to $k$ ). Here, $v_{0}, v_{1}$ are supposed to be in $\{0,1\}^{k}$.

- (Binding) For any adversary $\mathcal{S}^{*}$ and any tag, the probability

$$
\begin{aligned}
\operatorname{Pr}[\sigma & \leftarrow \mathcal{K}\left(1^{k}\right),\left(\left(d_{1}, d_{2}\right), c\right) \leftarrow\left\langle\mathcal{S}^{*}(\sigma, \text { tag }), \mathcal{R}_{\sigma, t a g}\right\rangle,\left(-, v_{1}\right) \leftarrow\left\langle\mathcal{S}^{*}\left(d_{1}\right), \mathcal{R}_{\sigma, t a g}(c)\right\rangle, \\
\left(-, v_{2}\right) & \left.\leftarrow\left\langle\mathcal{S}^{*}\left(d_{2}\right), \mathcal{R}_{\sigma, t a g}(c)\right\rangle: v_{1} \neq \perp, v_{2} \neq \perp, v_{1} \neq v_{2}\right]
\end{aligned}
$$

is negligible (with respect to $k$ ).

For our purpose, we need a new type of binding property, determining property. 
Definition 2 (Determining Property). A commitment scheme $\Sigma=(\mathcal{K}, \mathcal{S}, \mathcal{R})$ is said to be determining if there exists a deterministic function $\operatorname{det}(\cdot)$ (that depends only on $\Sigma$ and may not be efficiently computable) and for any feasible adversary $\mathcal{S}^{*}$ the probability

$$
\operatorname{Pr}\left[\sigma \leftarrow \mathcal{K}\left(1^{k}\right),(-, c) \leftarrow\left\langle\mathcal{S}^{*}(\sigma), R_{\sigma, t a g}\right\rangle: \exists d, r \text { s.t. }(-, v)=\left\langle\mathcal{S}^{*}(d), R_{\sigma, t a g}(c ; r)\right\rangle \Rightarrow v=\operatorname{det}(c)\right]
$$

is negligibly close to 1 (with respect to $k$ ). Here, tag is being chosen by $\mathcal{S}^{*}$. We call the unique value $v=\operatorname{det}(c)$ determining value of $c$.

In the above definition we note that the $d$ and $r$ are not supposed to be efficiently computable. The determining property means that any commitment $c$ generated by feasible adversaries $S^{*}$ must statistically determine the value $v$ that can be committed to under $c$. If $\Sigma$ is statistically binding then it is determining, and if $\Sigma$ is determining then it is computationally binding ${ }^{2}$. In the rest of paper, we only consider commitment schemes that are determining.

\subsection{Definition of Adaptive-Deniable-Concurrent Non-malleability}

Intuitively, we call a commitment scheme adaptive-deniable-concurrent non-malleable if the scheme permits no fake commitments even by a man-in-the-middle adversary that is able to invoke any number of left and right parties concurrently to receive/make commitments and is able to adaptively corrupt any number of left parties that complete commitment phases, and in addition if the scheme leaves no evidence of protocol execution.

Global CRS-KR model. To achieve the property in an efficient way, we rely on a global setup model, called global CRS-KR model, which is a simple extension of the KR model of [3]. In the global CRS-KR model, as in Figure 1, parties must register their identities to receive their public keys and a global CRS. Secret keys are sent only to corrupt participants.

\section{Functionality $\mathcal{G}_{\mathrm{crs}-k r}^{\mathrm{GenCRS}, \text { GenKey }}$}

Initialization Compute $\sigma \leftarrow$ GenCRS.

Registration Whenever activated by a party $P$ with id $I D$, if its key-pair is not yet registered, generate a key-pair $\left(p k_{I D}, s k_{I D}\right) \leftarrow$ GenKey. Return $\sigma$ and $p k_{I D}$ to $P$.

Retrieval Upon receipt of a retrieve request for id $I D$ from a party $P$, return $p k_{I D}$ to $P$. If the party $P$ has $I D$ as its own id and is corrupt, return also $s k_{I D}$ to $P$.

Fig. 1. The Global CRS-KR model.

For contrast, here we see the augmented CRS model of Canetti, Dodis, Pass and Walfish [5], in Figure 2 .

In the augmented CRS model, the public value is the same for any parties. However it needs different secret keys for every corrupt parties, and thus needs a master secret. Meanwhile, in the global CRS-KR model, although the public value depends on parties, it does not need any kind of master secret.

\footnotetext{
${ }^{2}$ If a commitment scheme is not computationally binding, then the $d$ must be efficiently computable by commitmentphase $S^{*}$ and $\operatorname{det}(c)$ must be ambiguous, meaning that the scheme is not determining.
} 


\section{Functionality $\mathcal{G}_{\text {acrs }}^{\text {Setup Extract }}$}

Initialization Compute $p k \leftarrow \operatorname{Setup}(m s k)$ for a random secret $m s k$.

Public value Whenever activated by any party with a request for the public key, return $p k$.

Retrieval Upon receipt of a retrieve request from a corrupt party $P$ with id $I D$, return $s k_{I D} \leftarrow$ Extract $(p k, I D, m s k)$ to $P$.

Fig. 2. The Augmented CRS model.

Thus, we cannot say any one is better than the another, unconditionally. The augmented CRS model is better in the view of efficiency of public values, and the global CRS-KR model is better in the cost of needed trust for the third party that implements the functionality, since the model does not need any master secret.

Note that, in both of the above two models, an adversary is supposed to corrupt some party, to participate a protocol-session as an active player. This means that an adversary must register its id and get the secret key of corrupt party that it plays.

The Definition. We define two experiments, as to a commitment scheme $\Sigma$ and an adversary $A$, as in Figure 3, in order to define adaptive-deniable-concurrent non-malleability of $\Sigma$.

- Experiment $\operatorname{mim}_{A}^{\Sigma}\left(v_{1}, \cdots, v_{m}, z\right)$ :

1. Invoke $A$ on input $z$. $A$ outputs a string $I D^{*}$ to register itself and tags $t_{1}, \ldots, t_{m}$ for $m$ left interactions.

2. Generate CRS $\sigma$ and a public/private-key pair $p k_{I D^{*}}, s k_{I D^{*}}$ for $I D^{*}$ as specified by $\Sigma$.

3. Receiving $\sigma, p k_{I D^{*}}, s k_{I D^{*}}, A$ carries over a concurrent man-in-the-middle attack: Each of $m$ left honest parties concurrently commits to $v_{i}$ with tag $t_{i}$ according to $\Sigma$ through transcript $c_{i}$ for $A(i=1, \ldots, m)$ and at the same time $A$ concurrently commits to some values with tags $t_{j}^{*}$ (chosen by $A$ ) through transcripts $c_{j}^{*}$ for each of $n$ right honest parties $(j=1, \ldots, n)$ with some polynomial $n=n(k)$. (If necessary, $A$ can receive public keys $p k_{I D}$ of any $I D$ it wants.)

4. The experiment outputs $\left(\right.$ view $\left._{A}, v_{1}^{*}, \ldots, v_{n}^{*}, d_{1}, \ldots, d_{m}\right)$. Here, view $w_{A}$ denotes the view of $A$ in the above attack, $v_{j}^{*}$ denotes the determining value $\operatorname{det}\left(c_{j}^{*}\right)$ of the right commitments $c_{j}^{*}(j=1, \ldots, n)$ and $d_{i}$ denotes honest decommitment of the left commitments $c_{i}$ (to $\left.v_{i}\right)(i=1, \ldots, m)$. However here, for each $j \in\{1, \ldots, n\}$, if $t_{j}^{*}=t_{i}$ with some $i$, then $v_{j}^{*}$ be overwritten to $\perp$.

- Experiment $\operatorname{sta}_{S}^{\Sigma}\left(v_{1}, \cdots, v_{m}, z\right)$ :

1. Invoke $S$ on input $z$. $S$ outputs a string $I D^{*}$ to register itself and tags $t_{1}, \ldots, t_{m}$.

2. Generate CRS $\sigma$ and a public/private-key pair $p k_{I D^{*}}, s k_{I D^{*}}$ of $I D^{*}$ as specified by $\Sigma$.

3. Receiving $\sigma, p k_{I D^{*}}, s k_{I D^{*}}, S$ concurrently commits to some values using tags $t_{j}^{*}$ (chosen by $S$ ) according to $\Sigma$ through transcripts $c_{j}^{*}$ for each of $n$ receivers with some polynomial $n=n(k)(j=1, \ldots, n)$ and outputs a simulated view view $A$ of $A$. (If necessary, $S$ can receive public keys $p k_{I D}$ of any $I D$ it wants.)

4. $S$ is given the values $v_{1}, \cdots, v_{m}$. Then, $S$ outputs decommitment $d_{i}$ (to $v_{i}$ ) that corresponds to the simulated commitments $c_{i}$ in the simulated view view $w_{A}(i=1, \ldots, m)$.

5. The experiment outputs $\left(\right.$ view $\left._{A}, v_{1}^{*}, \ldots, v_{n}^{*}, d_{1}, \ldots, d_{m}\right)$. Here, $v_{j}^{*}$ denotes the determining value $\operatorname{det}\left(c_{j}^{*}\right)$ of the right commitments $c_{j}^{*}(j=1, \ldots, n)$.

Fig. 3. Experiments $\operatorname{mim}_{A}^{\Sigma}$ and $\operatorname{sta}_{S}^{\Sigma}$.

Definition 3. A commitment scheme $\Sigma=(K, \mathcal{S}, \mathcal{R})$ is said to be adaptive-deniable-concurrent non-malleable in the global CRS-KR model if for any feasible adversary $A$, there exists a feasible algorithm $S$ and the ensemble $\left\{\operatorname{sta}_{S}^{\Sigma}\left(v_{1}, \cdots, v_{m}, z\right)\right\}_{v_{1}, \cdots, v_{m}, z}$ is computationally indistinguishable 
from the ensemble $\left\{\operatorname{mim}_{A}^{\Sigma}\left(v_{1}, \cdots, v_{m}, z\right)\right\}_{v_{1}, \cdots, v_{m}, z}$ with any polynomial $m=m(k)$, any values $v_{1}, \cdots, v_{m}\left(\in\{0,1\}^{k}\right)$ and any string $z$.

Note that Definition 3 incorporates the global CRS-KR model. An adversary needs to register its id $I D^{*}$ to mount an attack.

Basically, Definition 3 is in the similar line to the definition of concurrent non-malleability of [22, $18,20]$. In the first experiment $\operatorname{mim}_{A}^{\Sigma}$, a man-in-the-middle adversary $A$ carries over a concurrent man-in-the-middle attack between $m$ left parties and $n$ right parties. The resulting $A$ 's view and the determining values $v_{j}^{*}$ of right commitments must be simulated by the stand-alone simulator $S$ in the second experiment $\operatorname{sta}_{S}^{\Sigma}$. This means that commitments by left parties are no use for $A$ to make right commitments, i.e., concurrent non-malleability.

As seen above, in the second experiment, the simulator $S$ has to simulate left commitments in the simulated view of $A$. After completing such simulated left commitments as well as real right commitments, the simulator $S$ is given the values $v_{i}$. This models the situation of adaptive corruption of left honest parties, where left honest parties are corrupt just after completing commitments and the committed values are given to the adversary. Definition 3 requires that the simulator $S$ must be able to adapt decommitment of those simulated left commitments to those newly given values, i.e., adaptivity.

Moreover, note that the simulator $S$ is not capable to simulate the CRS $\sigma$ in the second experiment. The $\sigma$ is merely a given string also for $S$. This brings us deniability. (Recall that it is the key that the simulator is able to program CRS in the usual local CRS model. But this violated the deniability.)

Thus, Definition 3 concerns concurrent non-malleability, adaptivity and deniability of commitment schemes, all at once. (Note that we are not defining each of those three property, separately.)

Remark 1. In the above definition, adversary $A$ is restricted to be a type of "selective-tag", namely, $A$ is supposed to choose left tags $t_{1}, \ldots, t_{n}$ before it knows the CRS and $A$ must use different tags $t_{j}^{*}$ from $t_{i}$ for his challenges. The restriction is not restrictive. In fact, a tag-based commitment scheme that is secure for selective-tag-type adversaries is easily transformed into an ordinal commitment scheme without tags that is secure for fully adaptive adversaries, by using a method of the CHK transformation [8], just as in the cases of encryption schemes. (Sender generates a key-pair of strong one-time signature. It uses the verification key as a tag and signs a transcript by the corresponding secret key.)

\subsection{Definition of Straight-line Equivocal-Extractability}

As a more-easy-to-prove sufficient condition for the adaptive-deniable-concurrent non-malleability, we define straight-line equivocal-extractability of commitment schemes, that involves only a "classical" (instead of concurrent) man-in-the-middle adversary. The straight-line equivocal-extractability requires that a left party can be adaptively simulated by some feasible algorithm EQV that knows adversary's secret key and at the same time the property requires that the value committed to by the adversary for a right party can be extracted by some feasible algorithm EXT that knows the trapdoor of the global CRS. More formally, we define two experiments, as to a commitment scheme $\Sigma$ and an adversary $A$, as in Figure 4. 
- Experiment realCom ${ }_{A}^{\Sigma}(v, z)$ :

1. Invoke $A$ on input $z$. $A$ outputs a string $I D^{*}$ to register itself and a tag $t$ for the left interaction.

2. Generate CRS $\sigma$ and a public/private-key pair $p k_{I D^{*}}, s k_{I D^{*}}$ of $I D^{*}$ as specified by $\Sigma$.

3. Receiving $\sigma, p k_{I D^{*}}, s k_{I D^{*}}, A$ carries over a (classical) man-in-the-middle attack: A left honest party commits to $v$ with tag $t$ according to $\Sigma$ through transcript $c$ for $A$ and at the same time $A$ commits to some value using tag $t^{*}$ (chosen by $A$ ) through transcript $c^{*}$ for a right honest party. (If necessary, $A$ can receive public keys $p k_{I D}$ of any $I D$ it wants.)

4. The experiment outputs $\left(\tau\right.$, view $\left._{A}, v^{*}, d\right)$. Here, $\tau$ denotes the trapdoor of CRS $\sigma$, view $w_{A}$ denotes the view of $A$ in the above attack, $v^{*}$ denotes the determining value $\operatorname{det}\left(c^{*}\right)$ of the right commitment $c^{*}$ and $d$ denotes honest decommitment of the left commitment $c$ (to $v$ ). However here, if $t^{*}=t$ then $v^{*}$ be overwritten to $\perp$.

- Experiment fakeCom ${ }_{\mathrm{EQV}, A, \mathrm{EXT}}^{\Sigma}(v, z)$ :

1. Invoke $A$ on input $z$. $A$ outputs a string $I D^{*}$ to register itself and a tag $t$ for the left interaction.

2. Generate CRS $\sigma$ with trapdoor $\tau$ and a public/private-key pair $p k_{I D^{*}}, s k_{I D^{*}}$ of $I D^{*}$ as specified by $\Sigma$.

3. Receiving $\sigma, p k_{I D^{*}}, s k_{I D^{*}}, A$ carries over a (classical) man-in-the-middle attack with a dummy left party: The algorithm EQV, on input $t, s k_{I D^{*}}$, plays the role of honest left party and commits to a dummy value $w$ with tag $t$ according to $\Sigma$ through transcript $c$ for $A$ and at the same time $A$ commits to some value using tag $t^{*}$ (chosen by $A$ ) through transcript $c^{*}$ for a right honest party. (If necessary, $A$ can receive public keys $p k_{I D}$ of any $I D$ it wants.) Let $d^{\prime}$ be a local output of $\operatorname{EQV}\left(t, s k_{I D^{*}}, w\right)$.

4. If $t^{*}=t$ then set $v^{*}$ to be $\perp$. Otherwise, invoke algorithm EXT on input the trapdoor $\tau$ of CRS $\sigma$ and the view view $A$ of $A$, and set its output to $v^{*}$.

5. Invoke (the second stage of) algorithm EQV on input $d^{\prime}$ and $v$, and get its output $d$.

6. The experiment outputs $\left(\tau\right.$, view $\left._{A}, v^{*}, d\right)$.

Fig. 4. Experiments realCom ${ }_{A}^{\Sigma}$ and fakeCom ${ }_{\mathrm{EQV}, A, \mathrm{EXT}}^{\Sigma}$.

Definition 4. A commitment scheme $\Sigma=(K, \mathcal{S}, \mathcal{R})$ is said to be straight-line equivocal-extractable in the global CRS-KR model if there exists a feasible algorithm EQV such that for any feasible adver-

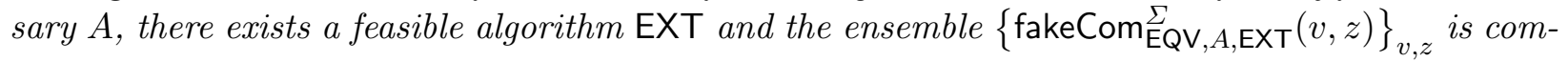
putationally indistinguishable from the ensemble $\left\{\operatorname{realCom}_{A}^{\Sigma}(v, z)\right\}_{v, z}$ with any value $v\left(\in\{0,1\}^{k}\right)$ and any string $z$.

Note that Definition 4 incorporates the global CRS-KR model. An adversary needs to register its id $I D^{*}$ to mount an attack.

Remark 2. The definition requires that the algorithm EQV is independent of adversaries $A$.

Theorem 1. If a commitment scheme $\Sigma=(K, \mathcal{S}, \mathcal{R})$ is determining, public-coin for $\mathcal{R}$ and straight-line equivocal-extractable in the global CRS-KR model, then $\Sigma$ is adaptive-deniable-concurrent non-malleable in the global CRS-KR model.

Proof Idea. To prove the theorem, we construct a stand-alone man-in-the-middle adversary $A_{i j}$ from an assumed concurrent adversary $A$ against the scheme, that internally simulates left parties besides the $i$-th left party and right parties besides the $j$-th right party for $A$. We use the straightline equivocal-extractability for $A_{i j}$ to upper bound advantage of the assumed $A$, using a hybrid argument. In doing that, as one subtle point, we need to efficiently construct the view of $A_{i 2}, \ldots, A_{i m}$ given the view of $A_{i 1}$, that will be possible since the scheme is public-coin for receiver $\mathcal{R}$.

Proof. Let $\Sigma=(K, \mathcal{S}, \mathcal{R})$ be a commitment scheme that is determining, public-coin for $\mathcal{R}$ and straight-line equivocal-extractable. Let EQV be the algorithm that is guaranteed to exist by the definition of straight-line equivocal-extractability for $\Sigma$. Let $A$ be arbitrary feasible adversary against 
$\Sigma$. We can assume $A$ is deterministic without loss of generality (by supposing $A$ 's coins are included in its input $z$ ). We want to construct a simulator $S$ that satisfies

$$
\operatorname{sta}_{S}^{\Sigma}\left(v_{1}, \cdots, v_{m}, z\right) \equiv_{c} \operatorname{mim}_{A}^{\Sigma}\left(v_{1}, \cdots, v_{m}, z\right)
$$

for any values $v_{1}, \cdots, v_{m}\left(\in\{0,1\}^{k}\right)$ and any string $z$. We construct such simulator $S$ as follows:

- Simulator $S$ on input a string $z$ :

1. $S$ invokes $A$ on input $z$. $A$ outputs $I D^{*}, t_{1}, \ldots, t_{m}$. Then, $S$ also outputs $I D^{*}, t_{1}, \ldots, t_{m}$.

2. $S$ receives CRS $\sigma$ and a public/private-key pair $p k_{I D^{*}}, s k_{I D^{*}}$. Giving those $\sigma, p k_{I D^{*}}, s k_{I D^{*}}$ to $A, S$ plays each of $m$ left parties for $A$ by invoking EQV on $t_{i}, s k_{I D^{*}}, w$ (Here, $w$ is any string such as $\left.0^{k}\right)(i=1, \ldots, m)$. $S$ forwards messages of $A$ to each of $n$ right parties to its own outside right parties $\mathcal{R}_{\sigma, t_{j}^{*}}$ and forwards messages from $\mathcal{R}_{\sigma, t_{j}^{*}}$ to $A$ as its $j$-th right party messages $(j=1, \ldots, n)$. Let $d_{i}^{\prime}$ be the local output of $\operatorname{EQV}\left(t_{i}, s k_{I D^{*}}, w\right)(i=1, \ldots, m)$.

3. On halting $A, S$ outputs its view view $A$.

4. Receiving left values $v_{i}, S$ invokes the corresponding second stage of EQV on $d_{i}^{\prime}$ and $v_{i}$ to get its output $d_{i}$ and outputs $d_{i}$ as its own output $(i=1, \ldots, m)$.

Toward showing Equation (1) with the above $S$, we first construct a (classical) man-in-themiddle adversary $A_{i, j}$ using $A$ as follows $(i=0, \ldots, m-1, j=1, \ldots, n)$.

- A man-in-the-middle adversary $A_{i, j}$ on input $z, v_{i+2}, \ldots, v_{m}$ :

1. $A_{i, j}$ internally invokes $A$ on input $z$. $A$ outputs $I D^{*}, t_{1}, \ldots, t_{m}$. Then, $A_{i, j}$ outputs $I D^{*}, t_{i+1}$.

2. Receiving CRS $\sigma$ and a public/private-key pair $p k_{I D^{*}}, s k_{I D^{*}}, A_{i, j}$ carries over a man-in-themiddle attack against its (outside) left party and right party, using the internally simulated $A$ that is given those $\sigma, p k_{I D^{*}}, s k_{I D^{*}}$.

(a) In the attack, $A_{i, j}$ internally simulates $m$ left parties for $A$ as follows.

i. $A_{i, j}$ simulates each of the first $i$ left parties by using $\alpha$-th copy of EQV on $t_{\alpha}, s k_{I D^{*}}, w$ $(\alpha=1, \ldots, i)$. ( $w$ is any dummy string.)

ii. $A_{i, j}$ forwards messages from its left party to $A$ as messages of $A$ 's $(i+1)$-th left party, and forwards messages from $A$ to its $(i+1)$-th left party to its left party.

iii. $A_{i, j}$ simulates the rest of $m-i-1$ left parties honestly to commit to $v_{\beta}(\beta=$ $i+2, \ldots, m)$.

(b) At the same time $A_{i, j}$ internally simulates $n$ right parties for $A$ as follows.

i. $A_{i, j}$ honestly simulates each of $n$ right parties except the $j$-th right party for $A$.

ii. $A_{i, j}$ forwards messages from its right party to $A$ as messages of $A$ 's $j$-th right party, and forwards messages from $A$ to $A$ 's $j$-th right party to its right party.

Using the above $A_{i, j}$, we define hybrid experiments hyb- $i_{A}^{\Sigma}\left(v_{1}, \ldots, v_{m}, z\right)$ for $i=0,1, \ldots, m$ as in Figure 5. In the experiments, when $i=m$, all of the left parties are simulated by EQV and hyb $-m_{A}^{\Sigma}$ is exactly distributed as $\operatorname{sta}_{S}^{\Sigma}$ :

$$
\operatorname{hyb}-m_{A}^{\Sigma}\left(v_{1}, \ldots, v_{m}, z\right) \equiv \operatorname{sta}_{S}^{\Sigma}\left(v_{1}, \cdots, v_{m}, z\right) .
$$

On the other hand, when $i=0$, all of the left parties are honestly simulated to commit to $v_{i}$. Moreover the straight-line equivocal-extractability of $\Sigma$ means that $\operatorname{det}\left(c_{j}^{*}\right)=\operatorname{EXT}^{(i, j)}\left(\tau, v i e w_{A_{i, j}}\right)$ (with exception of negligible probability). Hence, we have

$$
\operatorname{hyb}-0_{A}^{\Sigma}\left(v_{1}, \ldots, v_{m}, z\right) \equiv_{s} \operatorname{mim}_{A}^{\Sigma}\left(v_{1}, \cdots, v_{m}, z\right) \text {. }
$$


- Experiment hyb- $i_{A}^{\Sigma}\left(v_{1}, \ldots, v_{m}, z\right)$ :

1. Invoke $A$ on input $z$, that outputs a string $I D^{*}$ to register itself and tags $t_{1}, \ldots, t_{m}$ for left interactions.

2. Generate CRS $\sigma$ with trapdoor $\tau$ and a public/private-key pair $p k_{I D^{*}}, s k_{I D^{*}}$, and give them to $A$.

3. A begins a concurrent man-in-the-middle attack. Then,

(a) Simulate each of first $i$ left parties by $\alpha$-th copy of EQV on $t_{\alpha}, s k_{I D^{*}}, w(\alpha=1, \ldots, i)$.

(b) Simulate honestly each of the rest $m-i$ left parties to commit to $v_{\beta}(\beta=i+1, \ldots, m)$.

(c) Receiving these $m$ left commitments $c_{1}, \ldots, c_{m}$ concurrently, $A$ commits to some values with tags $t_{j}^{*}$ (chosen by $A$ ) through transcripts $c_{j}^{*}$ for each of $n$ right honest parties concurrently $(j=1, \ldots, n)$ with some polynomial $n=n(k)$.

Let $d_{\alpha}^{\prime}$ be the local output of $\operatorname{EQV}\left(t_{\alpha}, s k_{I D}, w\right)$ for $\alpha=1, \ldots, i$ and $d_{\beta}^{\prime}$ be the honest decommitment corresponding to $c_{\beta}$ for $\beta=i+1, \ldots, m$.

4. Let $A_{i, j}$ be the man-in-the-middle adversary defined as above from this $A$. We note that the third-step attack of $A$ can be viewed as an attack of $A_{i, j}$ on input $z, v_{i+2}, \ldots, v_{m}$ that stands in the middle of the $(i+1)$-th left party and the $j$-th right party. Let $\mathrm{EQV}$ and $\mathrm{EXT}^{(i, j)}$ be feasible algorithms for the $A_{i, j}$ that are guaranteed to exist by straight-line equivocal-extractability of $\Sigma$. (Recall EQV does not depend on the adversary by definition.)

5. For $j=1, \ldots, n$, if $t_{j}^{*}=t_{i}$, set $v_{j}^{*}=\perp$, else invoke $\mathrm{EXT}^{(i, j)}$ on $\tau, v i e w_{A_{i, j}}$ and set $v_{j}^{*}$ to its output.

6. For $i=1, \ldots, m$, invoke EQV on $d_{i}^{\prime}$ and $v$, and set $d_{i}$ to its output.

7. The experiment outputs $\left(\right.$ view $\left._{A}, v_{1}^{*}, \ldots, v_{n}^{*}, d_{1}, \ldots, d_{m}\right)$.

Fig. 5. Hybrid Experiments hyb- $i_{A}^{\Sigma}$.

By Equations (2) and (3), Equation (1) follows from the next claim by a standard hybrid argument.

Claim. For $i=0, \ldots, m-1$, we have hyb- $i_{A}^{\Sigma}\left(v_{1}, \ldots, v_{m}, z\right) \equiv_{c} \operatorname{hyb}-(i+1)_{A}^{\Sigma}\left(v_{1}, \ldots, v_{m}, z\right)$.

Proof. We define two auxiliary experiments real ${ }_{A}^{\Sigma}$ and ideal $\left.\right|_{A} ^{\Sigma}$. The experiment $\operatorname{real}_{A}^{\Sigma}\left(v_{1}, \ldots, v_{m}, z\right)$ first constructs $B=A_{i, 1}$. Then, it computes $\left(\tau, v_{i e w_{B}}, v_{1}^{*}, d_{i+1}\right) \leftarrow \operatorname{realCom}_{B}^{\Sigma}\left(v_{i+1},\left(z, v_{i+2}, \ldots, v_{m}\right)\right)$, and returns $\left(\right.$ view $\left._{A}, v_{1}^{*}, \ldots, v_{n}^{*}, d_{1}, \ldots, d_{n}\right) \leftarrow \operatorname{Expand}\left(\tau\right.$, view $\left._{B}, v_{1}^{*}, d_{i+1},\left(v_{1}, \ldots, v_{m}\right)\right)$. The experiment ideal ${ }_{A}^{\Sigma}$ is the same as real ${ }_{A}^{\Sigma}$ except that it uses fakeCom ${ }_{\mathrm{EQV}, B, \mathrm{EXT}}^{\Sigma}$, instead of realCom ${ }_{B}^{\Sigma}$, to compute $\left(\tau\right.$, view $\left._{B}, v_{1}^{*}, d_{i+1}\right)$.

In the above, the procedure Expand reconstructs view of $A_{i, j}$ from the view of $B=A_{i, 1}$ and extracts values $v_{j}^{*}$ committed to by $A$ in right interactions using EXT with trapdoor $\tau$. More precisely, Expand proceeds as follows:

- Procedure Expand $\left(\tau\right.$, view $\left._{B}, v_{1}^{*}, d_{i+1},\left(v_{1}, \ldots, v_{m}\right)\right)$ :

1. For $j=2, \ldots, n$, do:

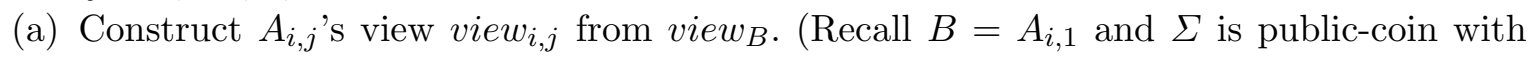
respect to receivers.)

(b) $v_{j}^{*} \leftarrow \operatorname{EXT}^{(i, j)}(\tau$, view $i, j)$ with the algorithm $\operatorname{EXT}^{(i, j)}$ for $A_{i, j}$.

2. For $\alpha=1, \ldots, i, i+2, \ldots, m$, do:

(a) Reconstruct from view vi $_{B}$ the local output $d_{\alpha}^{\prime}$ of the simulated $\alpha$-th left party. (Recall $B=A_{i, 1}$ internally simulates the $\alpha$-th left party.)

(b) $d_{\alpha} \leftarrow \operatorname{EQV}\left(d_{\alpha}^{\prime}, v_{\alpha}\right)$

3. Return $\left(\right.$ view $\left._{A}, v_{1}^{*}, \ldots, v_{n}^{*}, d_{1}, \ldots, d_{n}\right)$

It is easy to see that the view of $A$ (included in view $\left.w_{B}\right)$ in real ${ }_{A}^{\Sigma}\left(v_{1}, \ldots, v_{m}, z\right)$ is exactly distributed as $v i e w_{A}$ in hyb- $i_{A}^{\Sigma}$. (In both cases, the first $i$ left parties are simulated by EQV.) Then, 
by definition of Expand, we have

$$
\operatorname{hyb}-i_{A}^{\Sigma}\left(v_{1}, \ldots, v_{m}, z\right) \equiv \operatorname{real}{ }_{A}^{\Sigma}\left(v_{1}, \ldots, v_{m}, z\right) .
$$

Similarly, we have

$$
\text { hyb- }(i+1)_{A}^{\Sigma}\left(v_{1}, \ldots, v_{m}, z\right) \equiv \operatorname{ideal}_{A}^{\Sigma}\left(v_{1}, \ldots, v_{m}, z\right) .
$$

The straight-line equivocal-extractability of $\Sigma$ against $B$ means that the outputs of realCom ${ }_{B}^{\Sigma}$ and fakeCom $\sum_{\mathrm{EQV}, B, \mathrm{EXT}}^{\Sigma}$ are indistinguishable and then, since Expand is an efficient procedure, real ${ }_{A}^{\Sigma}\left(v_{1}, \ldots, v_{m}, z\right)$ and ideal ${ }_{A}^{\Sigma}\left(v_{1}, \ldots, v_{m}, z\right)$ are indistinguishable. The claim follows by Equation (4) and (5).

Remark 3. We can construct a deniable concurrent zero-knowledge argument if the adaptivedeniable-concurrent non-malleable commitment scheme once exists, by using the GMW protocol of graph 3-coloring instantiated with it. That means

- It is difficult (if not impossible) to construct adaptive-deniable-concurrent non-malleable commitment schemes only using the CRS model, since deniable concurrent zero-knowledge arguments are known to be difficult in the CRS model [21].

- An efficient construction of adaptive-deniable-concurrent non-malleable commitment scheme in the global CRS-KR model, that will be shown in the next section, gives an efficient deniable concurrent zero-knowledge argument in the global CRS-KR model. (We remark that the efficient concurrent zero-knowledge scheme of [12] in the auxiliary string is not deniable.)

\section{A Construction of Straight-line Equivocal-Extractable Commitment}

This section constructs a determining, public-coin for receivers, and straight-line equivocal-extractable commitment scheme in the global CRS-KR model, using bilinear groups. The scheme is practically efficient, using a constant number of pairing computations and three-round exchanges of linear-size messages.

\subsection{Design Principle}

The basic design of our commitment scheme follows the one of Damgård and Nielsen [14]. Generate a one-time commitment key of a base commitment using a coin-flipping protocol and then commit to a value by the base commitment with the generated one-time key. CRS is used in the coin-flipping.

However, in our scheme, the generated coins used to form a one-time commitment key are not opened to a receiver, remaining secret of a sender. To assure the coins are honestly generated and used to form one-time commitment key, the sender appends a non-interactive zero-knowledge argument for proving that honesty. (As seen later, the used NIZK argument can be constructed without using Cook reduction, depending on the property of the bilinear map.) More accurately, the argument proves that the sender formed the one-time commitment key honestly or the sender knows the receiver's secret key, that enables only simulator EQV to form a fake equivocal commitment.

The scheme "duplicates" some items in the sender messages so that extractor EXT can extract the generated coins (used to form a one-time commitment key) and values committed to by adversaries in the course of proving security with help of some KEA extractors. 


\subsection{Building Blocks}

The Homomorphic Commitment. As base commitment, we use the homomorphic commitment of Groth, Ostrovsky, Sahai [17]. The homomorphic commitment is built on a group $G$ with bilinear map. The commitment to a value $m$ is computed as $\operatorname{Com}(m ; r, t)=\left(M_{1}=g_{1}^{m} g_{4}^{r}, M_{2}=g_{2}^{m} g_{5}^{t}, M_{3}=\right.$ $\left.g_{3}^{m} g_{6}^{r+t}\right)$ with a tuple $\left(g_{1}, \ldots, g_{6}\right)$ of six elements in $G$ as a commitment key.

A tuple $\left(g_{1}, \ldots, g_{6}\right)$ is called linear when there exist $\alpha$ and $\beta$ that satisfy $g_{1}=g_{4}^{\alpha}, g_{2}=$ $g_{5}^{\beta}, g_{3}=g_{6}^{\alpha+\beta}$. A linear tuple $\left(g_{1}, \ldots, g_{6}\right)$ defines an equivocal (so perfectly hiding) commitment: $\operatorname{Com}\left(m_{0} ; r_{0}, t_{0}\right)=\operatorname{Com}\left(m ; r_{0}+\alpha\left(m_{0}-m\right), t_{0}+\beta\left(m_{0}-m\right)\right)$. On a while, a non-linear tuple $\left(g_{1}, \ldots, g_{6}\right)$ defines perfectly binding commitment. The commitment $\left(M_{1}, M_{2}, M_{3}\right)$ with a non-linear tuple $\left(g_{i}=g^{c_{i}}\right)$ determines its underlying value $m$ through $g^{m}=\left(M_{1}^{1 / c_{4}} M_{2}^{1 / c_{5}} M_{3}^{-1 / c_{6}}\right)^{1 /\left(c_{1} / c_{4}+c_{2} / c_{5}-c_{3} / c_{6}\right)}$.

The Perfect Non-interactive Zero-Knowledge Argument. To prove the above-mentioned honesty of generation of one-time commitment key, we incorporate the non-interactive zero-knowledge argument of Abe and Fehr [1] into our scheme in a following way.

Let CRS be a pair of elements $g, g_{c}$ in a group $G$ with bilinear map. A common input to a prover and a verifier is a triple of $A, b, \tilde{g}$ and a statement to be proved is: "There exist $a$ and $s$ satisfying that $A=g^{a} g_{c}^{s}$ and $\tilde{g}=g^{a b}$." The proof is simply a single element $P=g^{s}$, that is verified as $e\left(A, g^{b}\right)=e(\tilde{g}, g) e\left(P, g_{c}^{b}\right)$. The NIZKA is perfectly simulated in zero-knowledge, if one knows $e_{c}$ satisfying $g_{c}=g^{e_{c}}$, as $P=\left(A \tilde{g}^{-1 / b}\right)^{1 / e_{c}}$. The NIZKA is computationally sound, for example, under the Diffie-Hellman inversion assumption in a following way. Given an opening $(a, s)$ of $A=g^{a} g_{c}^{s}$, an opening $c$ of $\tilde{g}=g^{c}$ and a convincing proof $P$, one can efficiently compute $g^{1 / e_{c}}$ as $g^{1 / e_{c}}=\left(g^{-s} P\right)^{b /(a b-c)}$ if $a b \neq c$. Note that we need openings of $A$ and $\tilde{g}$ to use the soundness, that would require some use of KEA extractors, as in [1].

\subsection{The Commitment Scheme}

We describe our commitment scheme. Our scheme $\Sigma=\left(\Sigma^{\prime} \mid \Sigma^{\prime}\right)$ executes two parallel independent copies of subscheme $\Sigma^{\prime}=(K, S, R)$ in the global CRS-KR model. Each of these two executions uses an independent global CRS and an independent public/private key pair. If both executions of $\Sigma^{\prime}$ output a same value $m$, the scheme $\Sigma$ outputs that value $m$. (Otherwise, it outputs $\perp$.)

Let $G$ be a group of prime order $q$ with bilinear map $e(\cdot, \cdot): G \times G \rightarrow G_{T}$ and let $H$ be an injective function from $\{0,1\}^{\text {tagLen }}$ to $\mathbb{Z}_{q}$ (with length tagLen of tag strings). The subscheme $\Sigma^{\prime}=(K, \mathcal{S}, \mathcal{R})$ on group $G$ proceeds as in Figure 6 . $(\mathrm{DH}(\cdot, \cdot, \cdot, \cdot)$ means that a given tuple constitutes a DH-tuple.) It relies on a global CRS consisting of five elements $\left(g, g_{c}, g_{x}, h_{x}, g_{y}\right)$ in $G$ and requires the knowledge of receiver's public-key $g_{I D}$ that is also an element of $G$.

First, parties run a coin-tossing protocol using (part of) the CRS to generate random coins $c_{1}, \ldots, c_{6}$ in $\mathbb{Z}_{q}{ }^{*}$. Then, sender $\mathcal{S}$ forms a one-time commitment key $\left(g_{1}, \ldots, g_{6}\right)$ using the generated coins $c_{1}, \ldots, c_{6}$ as $g_{i}=g^{c_{i}}$, and computes NIZK arguments $P_{1}, \ldots, P_{6}$ that yield the one-time key $\left(g_{1}, \ldots, g_{6}\right)$ was honestly generated from $c_{1}, \ldots, c_{6}$ or $\mathcal{S}$ knows the secret key of $\mathcal{R}$. (Construction of the OR proof follows the standard way of making OR-proof of $\Sigma$ protocols [7].) In the course, some part of the CRS is used only after 'twisted' with the tag tag to prevent adversaries from copying generated coins from/to another sessions. Main body of commitment to input $m$ is generated with homomorphic commitment using that one-time key as $M_{1}=g_{1}{ }^{m} g_{4}{ }^{r}, M_{2}=g_{2}{ }^{m} g_{5}{ }^{t}, M_{3}=g_{3}{ }^{m} g_{6}{ }^{r+t}$. 
Generation of CRS: $K$ selects random four elements $e_{c}, e_{x}, d_{x}, e_{y}$ from $\mathbb{Z}_{q}$ and a random element $g$ of $G$. $K$ computes $g_{c}=g^{e_{c}}, g_{x}=g^{e_{x}}, h_{x}=g_{x}^{d_{x}}, g_{y}=g^{e_{y}}$ and outputs $\sigma=\left(g, g_{c}, g_{x}, h_{x}, g_{y}\right)$.

Commitment phase: Sender $\mathcal{S}$ commits to value $m\left(\in \mathbb{Z}_{q}\right)$ using tag tag and CRS $\sigma=\left(g, g_{c}, h_{c}, g_{x}, h_{x}\right)$ for receiver $\mathcal{R}$ with registered public key $g_{I D}\left(=g^{e_{I D}}\right)$, as follows:

1. $\mathcal{S}$ randomly chooses $a_{1}, \ldots, a_{6}, s_{1}, \ldots, s_{6}, b_{1}^{\prime}, \ldots, b_{6}^{\prime}$ from $\mathbb{Z}_{q}^{*}$ and $w_{1}, \ldots, w_{6}$ from $\mathbb{Z}_{q}$. For $i=1, \ldots, 6$, $\mathcal{S}$ computes

$$
A_{i}=g^{a_{i}} g_{c}^{s_{i}}, U_{i}=g^{w_{i}} g_{I D} b_{i}^{\prime} .
$$

$\mathcal{S}$ sends $A_{1}, \ldots, A_{6}, U_{1}, \ldots, U_{6}$ to $\mathcal{R}$

2. $\mathcal{R}$ randomly chooses $b_{1}, \ldots, b_{6}$ from $\mathbb{Z}_{q}^{*}$ and sends them to $\mathcal{S}$.

3. In response $\mathcal{S}$ computes, for $i=1, \ldots, 6$,

$$
b_{i}^{\prime \prime}=b_{i} / b_{i}^{\prime}, c_{i}=a_{i} b_{i}^{\prime \prime}, g_{i}=g^{c_{i}}, h_{i}=G_{x}{ }^{c_{i}}, P_{i}=g^{s_{i}}, Q_{i}=g_{y}{ }^{s_{i}}
$$

with $G_{x}=g_{x}^{H(\operatorname{tag})} h_{x}$. Then, $\mathcal{S}$ selects random elements $r, t$ from $\mathbb{Z}_{q}$ and computes

$$
M_{1}=g_{1}{ }^{m} g_{4}{ }^{r}, M_{2}=g_{2}{ }^{m} g_{5}{ }^{t}, M_{3}=g_{3}{ }^{m} g_{6}{ }^{r+t} \text {. }
$$

$\mathcal{S}$ sends the following items to $\mathcal{R}$,

$$
g_{1}, \ldots, g_{6}, h_{1}, \ldots, h_{6}, P_{1}, \ldots, P_{6}, Q_{1}, \ldots, Q_{6}, b_{1}^{\prime}, \ldots, b_{6}^{\prime}, w_{1}, \ldots, w_{6}, M_{1}, M_{2}, M_{3}
$$

4. $\mathcal{R}$ checks, for $i=1, \ldots, 6$,

$$
\operatorname{DH}\left(g, G_{x}, g_{i}, h_{i}\right), \operatorname{DH}\left(g, g_{y}, P_{i}, Q_{i}\right), U_{i}=g^{w_{i}} g_{I D} b_{i}^{\prime}, e\left(A_{i}, g^{b_{i}^{\prime \prime}}\right)=e\left(g_{i}, g\right) e\left(P_{i}, g_{c}^{b_{i}^{\prime \prime}}\right)
$$

with $b_{i}^{\prime \prime}=b_{i} / b_{i}^{\prime}$. If any of them (for any $i$ ) is not true, $\mathcal{R}$ aborts.

Decommitment phase: $\mathcal{S}$ sends $m, r, t$ to $\mathcal{R}$, who accepts it if $M_{1}=g_{1}{ }^{m} g_{4}{ }^{r}, M_{2}=g_{2}{ }^{m} g_{5}{ }^{t}, M_{3}=g_{3}{ }^{m} g_{6}{ }^{r+t}$.

Fig. 6. The Commitment Subscheme $\Sigma^{\prime}=(K, \mathcal{S}, \mathcal{R})$

In the scheme, several messages are duplicated such as $h_{1}, \ldots, h_{6}$ for $g_{1}, \ldots, g_{6}$ and $Q_{1}, \ldots, Q_{6}$ for $P_{1}, \ldots, P_{6}$. Those will be used by KEA-extractors to extract the coins generated by adversaries from its view in the proof of security. A decommitment is done in a canonical way.

As seen, the proposed scheme is practically efficient, using a constant number of pairing computations and three-round exchanges of linear-size messages.

\subsection{Security}

First, we review two necessary assumptions. Let $G$ denote a group of $k$-bit prime order $q$. The decisional linear assumption holds on $G$ if linear tuples $\left(g_{4}{ }^{\alpha}, g_{5}{ }^{\beta}, g_{6}{ }^{\alpha+\beta}, g_{4}, g_{5}, g_{6}\right)$ are computationally indistinguishable from random tuples $\left(g_{4}{ }^{\alpha}, g_{5}{ }^{\beta}, g_{6}{ }^{\gamma}, g_{4}, g_{5}, g_{6}\right)$ with random elements $g_{4}, g_{5}, g_{6}$ in $G$ and random $\alpha, \beta, \gamma$ in $\mathbb{Z}_{q}$.

The knowledge of exponent assumption [11] means that it is possible only if one knows $b$ to generate a pair $\left(g^{b}, g^{a b}\right)$ given a random $g^{a}$. More formally, for feasible algorithms $H, H^{*}$ and any string $w$, an experiment $\operatorname{Exp}_{G, H, H^{*}}^{w}$ is defined as follows. $H$ is given $q, g, A=g^{a}$ (with random $a$ from $\mathbb{Z}_{q}$ ) and $w$, and outputs $(B, W)$. On the same inputs $q, g, A$ and $w, H^{*}$ is invoked and outputs $b$. If $W=B^{a}$ and $B \neq g^{b}$, the experiment outputs 1 , otherwise outputs 0 . The knowledge of exponent assumption holds on $G$ if for any $w$ and any feasible adversary $H$ (called KEA-adversary) there exists a feasible $H^{*}$ (called KEA-extractor) with negligible advantage $\mathbf{A d v}_{G, H, H^{*}}^{w}(k):=\operatorname{Pr}\left[\operatorname{Exp}_{G, H, H^{*}}^{w}(k)=1\right]$. 
We begin to analyze security of the proposed commitment scheme $\Sigma=\left(\Sigma^{\prime} \mid \Sigma^{\prime}\right)$. In the analysis, we use ordinal characters such as $a, b, c, \ldots$ for items in one of the two parallel executions of the subscheme $\Sigma^{\prime}$ on which we have a current focus, and use characters with superscript + such as $a^{+}, b^{+}, c^{+}, \ldots$ for the corresponding items in the another parallel execution. First we see its computational hiding property.

Proposition 1. Under the decisional linear assumption on $G$, the proposed commitment scheme is computationally hiding in the global CRS-KR model.

Proof. Suppose that a feasible adversary $\mathcal{R}^{*}$ with identity $I D^{*}$ breaks the hiding property of the proposed scheme $\Sigma$ using tag tag with non-negligible probability.

Using such $\mathcal{R}^{*}$, we construct an efficient distinguisher $D$ that distinguishes between a pair of linear tuples and a pair of random tuples. Given a pair of tuples as input, $D$ simulates a sender for $\mathcal{R}^{*}$ and plugs the input tuple into the second sender-message as a one-time commitment key, for each of the two parallel executions of the subscheme $\Sigma^{\prime}$. In doing that, $D$ uses the knowledge of simulated secret key of $\mathcal{R}^{*}$, and proves it knows the secret key in the OR proof, instead of proving honesty of the fake one-time commitment key. More details follow.

Distinguisher $D$ on input $\left(\left(g_{1}, \ldots, g_{6}\right),\left(g_{1}^{+}, \ldots, g_{6}^{+}\right)\right)$:

1. (Simulate a sender for $\mathcal{R}^{*}$.) $D$ invokes $\mathcal{R}^{*}$ and does as follows, for each of the two parallel executions of the subscheme.

(a) (Emulate CRS and a key-pair.) $D$ selects random $e_{I D^{*}}$ from $\mathbb{Z}_{q}{ }^{*}$ and a random $g$ from $G$. $D$ sets $g_{I D^{*}}=g^{e_{I D^{*}}}$ and gives $\left(g_{I D^{*}}, e_{I D^{*}}\right)$ as her pair of public/private keys to $\mathcal{R}^{*}$. In addition, $D$ chooses random $e_{c}, e_{x}, d_{x}, e_{y}$ from $\mathbb{Z}_{q}$, sets $g_{c}=g^{e_{c}}, g_{x}=g^{e_{x}}, h_{x}=g_{x}^{d_{x}}, g_{y}=g^{e_{y}}$, and gives $\operatorname{tag}$ and $\left(g, g_{c}, g_{x}, h_{x}, g_{y}\right)$ to $\mathcal{R}^{*} . D$ records $\eta=e_{x} \cdot\left(H(t a g)+d_{x}\right)$ for later use. (Note $G_{x}=g^{\eta}$.)

(b) (Emulate the first message.) Receiving a pair of messages $\left(m_{0}, m_{1}\right)$ from $\mathcal{R}^{*}, D$ selects a random bit $b$ (only this process is common for the two parallel executions). Then, $D$ selects random $\left(s_{i}\right),\left(k_{i}\right),\left(b_{i}^{\prime \prime}\right)$ from $\mathbb{Z}_{q}$ and computes $A_{i}=g_{i}^{1 / b_{i}^{\prime \prime}} g_{c}^{s_{i}}$ (with $g_{i}$ in the input) and $U_{i}=g^{k_{i}}$ for $i=1, \ldots, 6$. $D$ sends $\left(A_{i}\right),\left(U_{i}\right)$ to $\mathcal{R}^{*}$.

(c) (Emulate the second message.) Receiving challenge $\left(b_{i}^{*}\right)$ from $\mathcal{R}^{*}$, to generate the second sender-message $\left(\left(g_{i}\right),\left(h_{i}\right), \ldots,\left(M_{i}\right)\right), D$ uses its own input $\left(g_{i}\right)$ (or $\left.\left(g_{i}^{+}\right)\right)$as the $\left(g_{i}\right)$ in the second message, computes $\left(h_{i}\right)$ as $h_{i}=g_{i}^{\eta}$ with $\eta$ recorded at Step 1a and honestly computes the proofs $P_{i}=g^{s_{i}}, Q_{i}=g_{y}{ }^{s_{i}}$. Then, with $b_{i}^{\prime}=b_{i}^{*} / b_{i}^{\prime \prime}, D$ computes $w_{i}=k_{i}-b_{i}^{\prime} e_{I D^{*}}$ using the secret key $e_{I D^{*}}$. Finally, $D$ computes $\left(M_{1}, M_{2}, M_{3}\right)$ as honest homomorphic commitment to $m_{b}$ with its own input $\left(g_{1}, \ldots, g_{6}\right)$ using as a commitment key. $D$ sends the second message $\left(\left(g_{i}\right),\left(h_{i}\right),\left(P_{i}\right),\left(Q_{i}\right),\left(b_{i}^{\prime}\right),\left(w_{i}\right),\left(M_{i}\right)\right)$ to $\mathcal{R}^{*}$.

2. (Output.) If $\mathcal{R}^{*}$ outputs $\hat{b}$ which is equal to $b$, then $D$ outputs 1 , otherwise outputs 0 .

We evaluate the probability that $D$ outputs 1 . When given both $\left(g_{1}, \ldots, g_{6}\right)$ and $\left(g_{1}^{+}, \ldots, g_{6}^{+}\right)$ are linear, the simulated view of $\mathcal{R}^{*}$ in $D$ is completely independent of $b$ by the perfect hiding property of homomorphic commitment with linear tuples. So, the probability that $D$ outputs 1 is $1 / 2$. 
Suppose both $\left(g_{1}, \ldots, g_{6}\right)$ and $\left(g_{1}^{+}, \ldots, g_{6}^{+}\right)$are random tuples. Let $c_{i}$ and $a_{i}$ be values defined by $g_{i}=g^{c_{i}}$ and $a_{i}=c_{i} / b_{i}^{\prime \prime}$. As for transcripts generated by $D$, we have

$$
\begin{aligned}
A_{i} & =g_{i}^{1 / b_{i}^{\prime \prime}} g_{c}^{s_{i}}=g^{c_{i} / b_{i}^{\prime \prime}} g_{c}^{s_{i}}=g^{a_{i}} g_{c}^{s_{i}} \\
U_{i} & =g^{k_{i}}=g^{w_{i}+b_{i}^{\prime} e_{I D^{*}}}=g^{w_{i}} g_{I D^{*}} b_{i}^{\prime} \\
g_{i} & =g^{c_{i}}, h_{i}=g_{i}^{\eta}=\left(g^{c_{i}}\right)^{\eta}=\left(g^{\eta}\right)^{c_{i}}=G_{x}^{c_{i}} \\
P_{i} & =g^{s_{i}}, Q_{i}=g_{y}^{s_{i}} .
\end{aligned}
$$

Thus, the simulated transcript is also determined by values $\left(a_{i}\right),\left(s_{i}\right),\left(w_{i}\right),\left(b_{i}^{\prime}\right),\left(c_{i}\right)$ just as the real transcripts. Here, by the description of $D$, we see $\left(c_{i}\right),\left(b_{i}^{\prime \prime}\right),\left(s_{i}\right),\left(k_{i}\right)$ among them are independently uniformly distributed and the rest $\left(a_{i}\right),\left(b_{i}^{\prime}\right),\left(w_{i}\right)$ are determined through the relations $c_{i}=a_{i} b_{i}^{\prime \prime}, b_{i}^{*}=b_{i}^{\prime} b_{i}^{\prime \prime}, w_{i}=k_{i}-b_{i}^{\prime} x_{I D}$ by them. On a while, in the real transcript, $\left(a_{i}\right),\left(b_{i}^{\prime}\right),\left(s_{i}\right),\left(w_{i}\right)$ are independently uniform and the remaining $\left(c_{i}\right),\left(b_{i}^{\prime \prime}\right),\left(k_{i}\right)$ are determined by the same relations by them. So, we see the distribution of $\left(a_{i}\right),\left(s_{i}\right),\left(w_{i}\right),\left(b_{i}^{\prime}\right),\left(c_{i}\right)$ is identical regardless whether it is simulated or real. Hence, the simulated view of $\mathcal{R}^{*}$ by $D$ is same as the real view of $\mathcal{R}^{*}$, and the probability that $D$ outputs 1 given random tuples $\left(g_{1}, \ldots, g_{6}\right)$ and $\left(g_{1}^{+}, \ldots, g_{6}^{+}\right)$is equal to the success probability of $\mathcal{R}^{*}$ to guess the committed values in the definition of hiding property. This must be nonnegligibly larger than $1 / 2$ by the contradictive assumption.

Hence, $D$ has non-negligible advantage to distinguish between a pair of linear tuples and a pair of random tuples. Such $D$, by standard argument, implies a distinguisher between a linear tuple and a random tuple with non-negligible advantage, contradicting to the decisional linear assumption.

Second, we show the determining property (Definition 2) of the proposed scheme.

Proposition 2. Under the discrete logarithm assumption and the knowledge of exponent assumption on $G$, the proposed commitment scheme is determining in the global CRS-KR model.

Proof. Since homomorphic commitment with non-linear tuples is perfectly binding, it is enough to show that any feasible adversarial sender $\mathcal{S}^{*}$ can generate linear one-time commitment keys $\left(g_{1}, \ldots, g_{6}\right)$ in (both of) the two parallel executions of the subscheme only with negligible probability.

Suppose a feasible adversary $\mathcal{S}^{*}$ generates a linear tuple $\left(g_{1}, \ldots, g_{6}\right)$ as its one-time commitment key in either of the two parallel executions of the subscheme $\Sigma^{\prime}$ for a honest receiver with identity $I D$ (using tag $t a g$ ) with non-negligible probability. Without loss of generality, we assume $\mathcal{S}^{*}$ generates a linear tuple in the second execution with non-negligible probability.

Using $\mathcal{S}^{*}$, we construct an efficient invertor $I$ that breaks the discrete-logarithm assumption on $G$ with help of some KEA-extractors. Given $g, g_{c}\left(=g^{e_{c}}\right), I$ sets $\left(g, g_{c}\right)$ in the corresponding part of CRS and simulates a receiver of $I D$ for internally invoked $\mathcal{S}^{*}$. In an execution of the subscheme, $I$ would receive the second sender-message $\left(g_{i}\right),\left(h_{i}\right),\left(P_{i}\right),\left(Q_{i}\right), \ldots$ from $\mathcal{S}^{*}$. If the messages passes the specified test by $\Sigma^{\prime}$, there should be some $c_{i}$ and $s_{i}$ that satisfy $\left(g_{i}, h_{i}\right)=\left(g^{c_{i}}, G_{x}{ }^{c_{i}}\right)$ and $\left(P_{i}, Q_{i}\right)=\left(g^{s_{i}}, g_{y}{ }^{s_{i}}\right)$. Then, $I$ can use some KEA-extractors $H_{c_{i}}^{*}$ and $H_{s_{i}}^{*}$ to extract such $c_{i}$ and $s_{i}$ respectively, that provides $I$ with an opening $a_{i}\left(=c_{i} b_{i}^{\prime} / b_{i}\right), s_{i}$ of $A_{i}=g^{a_{i}} g_{c}^{s_{i}}$ in the first sendermessage of $\mathcal{S}^{*}$. (For example, KEA-adversary $H_{c_{i}}$ on input $\left(g, g_{x}\right)$ reproduces the exact view of $\mathcal{S}^{*}$ invoked in $I$ using some auxiliary input $w_{x}$, and outputs $\left(g_{i}\left(=g^{c_{i}}\right), h_{i}^{1 /\left(H(t a g)+d_{x}\right)}\left(=g_{x}^{c_{i}}\right)\right)$. $)$ By rewinding $\mathcal{S}^{*}, I$ can obtain another opening $\hat{a}_{i}, \hat{s}_{i}$ of the same $A_{i}=g^{\hat{a}_{i}} g_{c}^{\hat{s}_{i}}$ from another second sender-message $\left(\hat{g}_{i}\right),\left(\hat{h}_{i}\right),\left(\hat{P}_{i}\right),\left(\hat{Q}_{i}\right), \ldots$ By the contradictive assumption we can suppose both $\left(g_{i}\right)$ 
and $\left(\hat{g}_{i}\right)$ are linear, and we will see it means that $a_{i} \neq \hat{a}_{i}$ with some $i$. Then $I$ can compute the desired discrete-log $e_{c}$ of $g_{c}$ over $g$, using such $a_{i}, s_{i}, \hat{a}_{i}, \hat{s}_{i}$ as $e_{c}=\left(\hat{a}_{i}-a_{i}\right)\left(s_{i}-\hat{s}_{i}\right)^{-1}$. More details follow.

Invertor $I$ on input $\left(g, g_{c}\left(=g^{e_{c}}\right)\right)$ :

1. (Simulate a receiver for $\mathcal{S}^{*}$.) $I$ invokes $\mathcal{S}^{*}$ and simulates an honest receiver for $\mathcal{S}^{*}$ as follows, for each of the two parallel executions of the subscheme.

(a) (First execution.) For the first execution, $I$ honestly generates CRS and public key for $\mathcal{S}^{*}$ and simulates the receiver in a completely honest way.

(b) (Second execution.) For the second execution, I simulates the receiver in a following way, using its input $\left(g, g_{c}\right)$ as part of CRS.

i. (Emulate a public/private-key pair and CRS.) $I$ selects a random $\delta$ from $\mathbb{Z}_{q}{ }^{*}$, sets $g_{I D}=g_{c}^{\delta}$ and gives $g_{I D}$ as receiver's public-key (for the second execution) to $\mathcal{S}^{*}$. $I$ also selects random $e_{x}, d_{x}, e_{y}$ from $\mathbb{Z}_{q}{ }^{*}$ under constraint that $H(t a g)+d_{x} \neq 0$ and sets $g_{x}=g^{e_{x}}, g_{y}=$ $g^{e_{y}}, h_{x}=g_{x}^{d_{x}}$ (with $g$ in the input). I gives $\left(g, g_{c}, g_{x}, h_{x}, g_{y}\right)$ as CRS (for the second execution) to $\mathcal{S}^{*}$, using $g, g_{c}$ in the input.

ii. (Emulate the challenge.) Receiving the first sender-message $\left(A_{i}\right),\left(U_{i}\right)$ from $\mathcal{S}^{*}, I$ takes random $\left(b_{i}\right)$ from $\mathbb{Z}_{q}{ }^{*}$ and sends them to $\mathcal{S}^{*}$.

iii. (Extract values generated by $\mathcal{S}^{*}$.) Receiving the second sender-message $\left(g_{i}\right), \ldots,\left(b_{i}^{\prime}\right), \ldots$ from $\mathcal{S}^{*}, I$ extracts values $\left(c_{i}\right),\left(a_{i}\right),\left(s_{i}\right)$ as follows, after verifying the equations specified by $\Sigma^{\prime}$.

A. (Extract $\left.\left(c_{i}\right)\right) I$ sets $w_{x}=\left(\delta, g_{c}, d_{x}, e_{y}, b_{1}, \ldots, b_{6}\right.$, coins1) (where coins1 denotes the coins used to honestly simulate the first execution) and for $i=1, \ldots, 6, I$ invokes KEA-extractor $H_{c_{i}}^{*}$ on input $\left(g, g_{x} ; w_{x}\right)$ and obtains the result $c_{i}$ to compute $a_{i}=$ $c_{i} b_{i}^{\prime} / b_{i}$. (Note that $e_{x}$ is independent of $w_{x}$.)

B. (Extract $\left.\left(s_{i}\right)\right) I$ sets $w_{y}=\left(\delta, g_{c}, e_{x}, d_{x}, b_{1}, \ldots, b_{6}\right.$, , coins1) and for $i=1, \ldots, 6, I$ invokes KEA-extractor $H_{s_{i}}^{*}$ on input $\left(g, g_{y} ; w_{y}\right)$ and obtains the result $s_{i}$. (Note that $e_{y}$ is independent of $w_{y}$.)

iv. (Rewind and extract new values.) $I$ rewinds $\mathcal{S}^{*}$ to Step 1(b)ii and repeats the processes with new challenge $\left(\hat{b}_{i}\right)$ to get the new extracted values $\left(\hat{c}_{i}\right),\left(\hat{a}_{i}\right),\left(\hat{s}_{i}\right)$, receiving another second message $\left(\hat{g}_{i}\right), \ldots,\left(\hat{b}_{i}^{\prime}\right), \ldots$ from $\mathcal{S}^{*}$.

2. (Compute the discrete log.) Finally,

(a) If there exists some $i$ satisfying $b_{i}^{\prime} \neq \hat{b}_{i}^{\prime}$, then $I$ computes the discrete-log $e_{c}$ using $w_{i}, b_{i}^{\prime}, \hat{w}_{i}, \hat{b}_{i}^{\prime}$ (as shown below).

(b) Otherwise if there exists some $i$ satisfying $a_{i} \neq \hat{a}_{i}$, then $I$ computes the discrete-log $e_{c}$ using $a_{i}, s_{i}, \hat{a}_{i}, \hat{s}_{i}$ (as shown below).

(c) Otherwise $I$ aborts.

It is direct to see $I$ perfectly simulates a real view of $\mathcal{S}^{*}$ using its input $\left(g, g_{c}\right)$ as part of CRS of the second execution. Then, $I$ invokes KEA-extractors $H_{c_{i}}^{*}$ at Step 1(b)iiiA and $H_{s_{i}}^{*}$ at Step 1(b)iiiB, respectively twice, by rewinding $\mathcal{S}^{*}$. From now on we condition ourselves on the nonnegligible event in which $\mathcal{S}^{*}$ generates a linear tuple $\left(g_{1}, \ldots, g_{6}\right)$ as its one-time commitment key in the second parallel execution, and completes the commitment phase. For a while, assume that

Claim 1 The extracted values $\left(c_{i}\right)$ (or $\left(\hat{c}_{i}\right)$ ) at Step 1(b)iiiA and $\left(s_{i}\right)$ (or $\left(\hat{s}_{i}\right)$ ) at Step 1(b)iiiB satisfy $g_{i}=g^{c_{i}}, P_{i}=g^{s_{i}}$ (or $\hat{g}_{i}=g^{\hat{c}_{i}}, \hat{P}_{i}=g^{\hat{s}_{i}}$ ) for $i=1, \ldots, 6$ with overwhelming probability. 
Under the claim, we can modify the right-hand side of the verifier equation as

$$
\begin{aligned}
e\left(A_{i}, g^{b_{i} / b_{i}^{\prime}}\right) & =e\left(g_{i}, g\right) e\left(P_{i}, g_{c}^{b_{i} / b_{i}^{\prime}}\right) \\
& =e\left(g^{c_{i}}, g\right) e\left(g^{s_{i}}, g_{c}^{b_{i} / b_{i}^{\prime}}\right)=e\left(g, g^{c_{i} b_{i}^{\prime} / b_{i}} g_{c}^{s_{i}}\right)^{b_{i} / b_{i}^{\prime}}
\end{aligned}
$$

that means $A_{i}=g^{c_{i} b_{i}^{\prime} / b_{i}} g_{c}^{s_{i}}=g^{a_{i}} g_{c}^{s_{i}}$ for $i=1, \ldots, 6$. Similarly, we have $A_{i}=g^{\hat{a}_{i}} g_{c}^{\hat{s}_{i}}$ for $i=1, \ldots, 6$.

Suppose there exists some $i$ satisfying $b_{i}^{\prime} \neq \hat{b}_{i}^{\prime}$ at Step $2 \mathrm{a}$. Then, by the verifier equation on $w_{i}$, we have $U_{i}=g^{w_{i}} g_{I D}^{b_{i}^{\prime}}=g^{\hat{w}_{i}} g_{I D}^{\hat{b}_{i}^{\prime}}$, that means $g_{I D}=g^{\left(\hat{w}_{i}-w_{i}\right)\left(b_{i}^{\prime}-\hat{b}_{i}^{\prime}\right)^{-1}}$. Then, since $g_{I D}=g_{c}^{\delta}, I$ can compute the desired discrete-log $e_{c}$ as $e_{c}=\left(\hat{w}_{i}-w_{i}\right)\left(b_{i}^{\prime}-\hat{b}_{i}^{\prime}\right)^{-1} \delta^{-1}$.

Else if there exists some $i$ satisfying $a_{i} \neq \hat{a}_{i}$ at Step $2 \mathrm{~b}$, since we have $A_{i}=g^{a_{i}} g_{c}^{s_{i}}=g^{\hat{a}_{i}} g_{c}^{\hat{s}_{i}}$ as seen above under Claim 1, it holds that $g_{c}=g^{\left(\hat{a}_{i}-a_{i}\right)\left(s_{i}-\hat{s}_{i}\right)^{-1}}$. Hence, $I$ can compute the discrete-log $e_{c}$ by $e_{c}=\left(\hat{a}_{i}-a_{i}\right)\left(s_{i}-\hat{s}_{i}\right)^{-1}$.

Otherwise, we have $b_{i}^{\prime}=\hat{b}_{i}^{\prime}$ and $a_{i}=\hat{a}_{i}$ for any $i$, then it must hold $\hat{c}_{i} / c_{i}=\hat{b}_{i} / b_{i}$ for any $i$. In this case, the probability that both tuples $\left(g_{i}\left(=g^{c_{i}}\right)\right)$ and $\left(\hat{g}_{i}\left(=g^{\hat{c}_{i}}\right)\right)$ are simultaneously linear is negligible (since the ratio $\hat{c}_{i} / c_{i}$ is uniformly random) and we see that $I$ reaches Step 2c only with negligible probability.

Thus, to complete the proof, all we need is to prove Claim 1.

Proof (of Claim 1). KEA-extractors $H_{c_{i}}^{*}$ correspond to the following KEA-adversaries $H_{c_{i}}$.

KEA-Adversary $H_{c_{i}}$ on input $\left(g, g_{x} ; w_{x}=\left(\delta, g_{c}, d_{x}, e_{y}, b_{1}, \ldots, b_{6}\right.\right.$, coins 1$\left.)\right)$ :

1. (Reproduce the view of $\mathcal{S}^{*}$.) $H_{c_{i}}$ invokes $\mathcal{S}^{*}$ and reproduces the view of $\mathcal{S}^{*}$ using $w_{x}$ as follows, for each of the two parallel executions.

(a) (First execution.) $H_{c_{i}}$ takes coins1 in $w_{x}$ and uses it to honestly simulate the first execution of the subscheme.

(b) (Second execution.) For the second execution, $H_{c_{i}}$ simulates the receiver in a following way. i. (Emulate a public/private-key pair and CRS.) $H_{c_{i}}$ takes $\delta$ and $g_{c}$ from its auxiliary input $w_{x}$ to regenerate $g_{I D}=g_{c}^{\delta}$ and gives it as receiver's public-key (for the second execution) to $\mathcal{S}^{*} . H_{c_{i}}$ takes $d_{x}, e_{y}$ from $w_{x}$ to regenerate $h_{x}=g_{x}^{d_{x}}, g_{y}=g^{e_{y}}$ and gives $\left(g, g_{c}, g_{x}, h_{x}, g_{y}\right)$ as CRS to $\mathcal{S}^{*}$.

ii. (Emulate the challenge.) Receiving the first sender-message $\left(A_{i}\right),\left(U_{i}\right)$ from $\mathcal{S}^{*}, H_{c_{i}}$ takes $\left(b_{i}\right)$ from $w_{x}$ and sends them to $\mathcal{S}^{*}$.

iii. (Generate an output.) Receiving the response $\left(g_{i}\right),\left(h_{i}\right), \ldots$ from $\mathcal{S}^{*}, H_{c_{i}}$ picks up $g_{i}$ and $h_{i}$ from it and outputs $\left(g_{i}, h_{i}^{1 /\left(H(\operatorname{tag})+d_{x}\right)}\right)$. (Recall $I$ generated $d_{x}$ so that $H(\operatorname{tag})+d_{x} \neq 0$.)

On input $\left(g, g_{x}\right), H_{c_{i}}$ reproduces the exact view of $\mathcal{S}^{*}$ invoked in $I$ using the auxiliary input $w_{x}$, and generates $\left(g_{i}, h_{i}\right)$ satisfying $\operatorname{DH}\left(g, G_{x}, g_{i}, h_{i}\right)$ with the same probability as $\mathcal{S}^{*}$ in $I$ does so. When $\left(g, G_{x}, g_{i}, h_{i}\right)$ is a DH-tuple and $g_{i}=g^{c_{i}}$, we have $h_{i}^{1 /\left(H(t a g)+d_{x}\right)}=G_{x}^{c_{i} /\left(H(t a g)+d_{x}\right)}=g_{x}^{c_{i}}$. (Here, recall that $I$ generated $d_{x}$ so that $H(\operatorname{tag})+d_{x} \neq 0$.) Thus $H_{c_{i}}$ outputs $\left(g^{c_{i}}, g_{x}^{c_{i}}\right)$ on input $\left(g, g_{x}\right)$. As directly verified, the items in $w_{x}$ are independent of the discrete-log $e_{x}$ of $g_{x}$ over $g$. Hence, by the knowledge of exponent assumption, we see that the corresponding KEA-extractor $H_{c_{i}}^{*}\left(\right.$ in $I$ ) outputs $c_{i}$ satisfying $g_{i}=g^{c_{i}}$ only with negligible exception (whenever $\mathcal{S}^{*}$ generates valid $\left.\left(g_{i}, h_{i}\right)\right)$.

KEA-adversary $H_{s_{i}}$ on input $\left(g, g_{y} ; w_{y}\right)$ proceeds as $H_{c_{i}}$ and outputs $\left(P_{i}=g^{s_{i}}, Q_{i}=g_{y}{ }^{s_{i}}\right)$. By a similar argument, we see $H_{s_{i}}^{*}$ in $I$ outputs $s_{i}$ satisfying $P_{i}=g^{s_{i}}$ only with negligible exception. 
Now we show the straight-line equivocal-extractability (Definition 4) of the proposed scheme.

Proposition 3. Under the decisional linear assumption and the knowledge of exponent assumption on $G$, the proposed commitment scheme is straight-line equivocal-extractable in the global CRS-KR model.

Proof Idea. To show the proposition, we need to construct a simulator EQV of a left party and an extractor EXT of values committed to by any feasible adversary $A$ for a right party, that satisfy Definition 4.

EQV is given $A$ 's secret key $e_{I D^{*}}$ and proves that it knows $e_{I D^{*}}$ in the second sender-message, instead of proving its honesty to the generation of one-time commitment key. By doing this, EQV can use a linear tuple as the one-time commitment key for its commitment. Since linear tuples define equivocal commitments, EQV is able to adaptively simulate left parties without knowing values actually being committed to. We show such simulation of left parties by EQV is indistinguishable from real left parties for feasible adversaries $A$ under the decisional linear assumption on $G$ with help of some KEA-extractors. (To show the indistinguishability, we need to extract the values committed to by $A$, that is enabled by using some KEA-extractors.)

EXT extracts the value committed to by $A$ from its view view $w_{A}$ using the trapdoor information of CRS. For that sake, EXT needs to extract the coins $c_{i}^{*}$, used by $A$ to generate its one-time commitment key. EXT invokes some KEA extractors on input $\left(g, g_{x}\right)$ using (some part of) the view view $w_{A}$ as auxiliary information to extract such coins $c_{i}^{*}$. Here, we need a care to use the knowledge of exponent assumption in a right way for such KEA-extractors, because the view view $w_{A}$ itself is dependent on the discrete-log between $g$ and $g_{x}$ that are included in CRS. (We can use the knowledge of exponent assumption only for KEA-extractors that uses auxiliary information that is independent of its input.) We will carefully examine distribution of view $w_{A}$ and pick up some portion from view $w_{A}$ that are independent of that discrete-log, making use of zero-knowledge simulators for proofs $P_{i}$.

In the course of the proof, one key point is to ensure that the adversary $A$ cannot use linear tuples as its own one-time commitment key, even $A$ receives linear tuples as one-time commitment keys from the simulated left party by EQV. This impossibility is brought by the use of tag tag in

the scheme for generation of $G_{x}=g_{x}^{H(t a g)} h_{x}$, that is in turn used for the generation of $\left(h_{i}\right)$ in the second sender-message. This dependency on tag prevents the flipped coins generated for one-time commitment keys from being copied from/to other sessions. The detailed proof is in Section A.

By Theorem 1, Proposition 2 and Proposition 3, we have

Theorem 2. Under the decisional linear assumption and the knowledge of exponent assumption on $G$, the proposed commitment scheme is adaptive-deniable-concurrent non-malleable in the global CRS-KR model.

\section{Conclusion}

We defined a notion of adaptive-deniable-concurrent non-malleable commitments, that captures the composability, deniability and adaptivity at once for commitment schemes. Then we defined a moreeasy-to-prove property of straight-line equivocal-extractability of commitment schemes and proved 
that it yields the adaptive-deniable-concurrent non-malleability in the global CRS-KR model. We also gave a construction of straight-line equivocal-extractable (especially, adaptive-deniableconcurrent non-malleable) commitment scheme in the global CRS-KR model, under the decisional linear assumption and the knowledge of exponent assumption on bilinear groups. The scheme is practically efficient, using a constant number of pairing computations and three-round exchanges of linear-size messages.

\section{References}

1. M. Abe and S. Fehr, Perfect NIZK with Adaptive Soundness, pp. 118-136, Proc. of TCC, LNCS 4392, 2007.

2. B. Barak, How to go beyond the black-box simulation barrier, Proc. 42nd FOCS, pp. 106-115, IEEE, 2001.

3. B. Barak, R. Canetti, J. B. Nielsen, R. Pass, Universally Composable Protocols with Relaxed Set-Up Assumptions, Proc. 45th FOCS, pp. 186-195, IEEE, 2004.

4. R. Canetti, Universally Composable Security: A New Paradigm for Cryptographic Protocols, pp. 136-145, Proc. of FOCS, 2001.

5. R. Canetti, Y. Dodis, R. Pass, S. Walfish, Universally Composable Security with Global Setup, pp. 61-85, Proc. of TCC 2007, LNCS 4392.

6. R. Canetti, M. Fischlin, Universally Composable Commitments, pp. 19-40, Proc. of Crypto, 2001.

7. R. Cramer, I. Damgård and B. Schoenmakers, Proofs of Partial Knowledge and Simplified Design of Witness Hiding Protocols, pp. 174-187, Proc. of Crypto 1994, LNCS 839.

8. R.Canetti, S.Halevi and J.Katz, Chosen-ciphertext security from identity-based encryption, EUROCRYPT 2004, LNCS 3027, pp.207-222, 2004.

9. G. D. Crescenzo, Y. Ishai and R. Ostrovsky, Non-interactive and Non-malleable Commitment, pp. 141-150, STOC 1998.

10. G. D. Crescenzo, J. Katz, R. Ostrovsky and A. Smith, Efficient and Non-interactive Non-malleable Commitments, pp. 40-59, Proc. of EuroCrypt 2001, LNCS 2045.

11. I. Damgård, Towards practical public-key cryptosystems provably-secure against chosen-ciphertext attacks, pp. 445-456, Proc. of CRYPTO '91, LNCS 576.

12. I. Damgård, Efficient Concurrent Zero-Knowledge in the Auxiliary String Model, pp. 418-430, Proc. of EUROCRYPT 2000, LNCS 1807.

13. I. Damgård and J. Groth, Non-interactive and reusable non-malleable commitment schemes, pp. 426-437, Proc. of STOC 2003, ACM Press.

14. I. Damgård and J. B. Nielsen, Perfect Hiding and Perfect Binding Universally Composable Commitment Schemes with Constant Expansion Factor, pp. 581-596, Proc. of Crypto 2002, LNCS 2442.

15. D. Dolev, C. Dwork and M. Naor, Non-Malleable Cryptography, SIAM Journal on Computing, Vol. 30(2), pp. 391-437, 2000.

16. M. Fischlin and R. Fischlin, Efficient Non-malleable Commitment Schemes, pp. 413-431, Proc. of Crypto 2000, LNCS 1880.

17. J. Groth, R. Ostrovsky, and A. Sahai, Perfect non-interactive zero knowledge for NP, 339-358, EUROCRYPT, LNCS 4004, 2006.

18. H. Lin, R. Pass, M. Venkitasubraamaniam, Concurrent Non-malleable Commitments from Any One-way Function, TCC 2008, LNCS 4948, pp. 571-588, 2008.

19. P. D. MacKenzie, M. K. Reiter and K. Yang, Alternatives to Non-malleability: Definitions, Constructions, and Applications, pp. 171-190, Proc. of TCC 2004, 2004.

20. R. Ostrovsky, G. Persiano, and I. Visconti, Simulation-Based Concurrent Non-malleable Commitments and Decommitments, TCC 2009, LNCS 5444, pp. 91-108, 2009.

21. R. Pass, On Deniabililty in the Common Reference String and Random Oracle Model, pp. 316-337, Proc. of CRYPTO 2003, LNCS 2729.

22. R. Pass, A. Rosen, Concurrent non-malleable commitments, 563 - 572, FOCS 2005.

\section{A Proof of Proposition 3}

Let $\Sigma=\left(\Sigma^{\prime} \mid \Sigma^{\prime}\right)$ with $\Sigma^{\prime}=(K, S, R)$ denote the proposed commitment scheme on $G$. We want to show there exists a feasible algorithm EQV such that for any feasible adversary $A$, there exists a 
feasible algorithm EXT and

$$
\text { fakeCom } \sum_{\mathrm{EQV}, A, \operatorname{EXT}}^{\Sigma}(m, z) \equiv_{c} \operatorname{realCom}_{A}^{\Sigma}(m, z)
$$

with any value $m\left(\in \mathbb{Z}_{q}\right)$ and any string $z$ (Definition 4$)$.

For that sake, we define four experiments $\mathbf{E x p}_{0}, \ldots, \mathbf{E x p}_{3}$ in the sequel and proves indistinguishability of their outputs step by step. $\mathbf{E x p}_{0}$ is nothing but the experiment realCom ${ }_{A}^{\Sigma}(m, z)$ instantiated with our $\Sigma$, and the final experiment $\mathbf{E x p}_{3}$ gives EQV and EXT, proving Equation (6).

$\mathbf{E x p}_{0}$. Given $m$ and $z$ as input, $\mathbf{E x p}_{0}$ invokes an adversary $A$ on $z$. First, $A$ selects a tag tag for the left interaction, its own id $I D^{*}$ and right-party's id $I D$. $A$ receives CRS $\sigma$, its public/privatekey pair $\left(g_{I D^{*}}, e_{I D^{*}}\right)$ and the right-party's public key $g_{I D}$, for each of the two parallel executions of the subscheme. Then, $\mathbf{E x p}_{0}$ simulates the left party that commits to $m$ and the right party for $A$, honestly. $\mathbf{E x p}_{0}$ returns trapdoor $\left(\tau, \tau^{+}\right)$of the CRSs of both executions, the simulated $A$ 's view view $w_{A}$, the determining value $m^{*}$ of $A$ 's commitment in view $w_{A}$ and left-party's decommitment $\left((m, r, t),\left(m, r^{+}, r^{+}\right)\right)$that corresponds to the left-party's commitment in view $A$. A full description of $\operatorname{Exp}_{0}$ is in Section B.

$\mathbf{E x p}_{1}$. Only the behavior of the left party is changed as follows. New left party proves the knowledge of $A$ 's secret key $e_{I D^{*}}$, instead of proving the honesty of the generation of the one-time commitment key. More precisely, for each of the two parallel executions of the subscheme, the new left party computes $U_{i}$ as $U_{i}=g^{k_{i}}$ with random $k_{i}$ (instead of $U_{i}=g^{w_{i}} g_{I D^{*}} b_{i}^{\prime}$ with $b_{i}^{\prime}$ selected in advance). Moreover, the left party selects a random one-time commitment key $\left(g_{i}=g^{c_{i}}\right)$ independently of $A_{i}=g^{a_{i}} g_{c}^{s_{i}}$ in the first message. Although proofs $P_{i}=g^{s_{i}}, Q_{i}=g_{y}{ }^{s_{i}}$ are honestly computed using $s_{i}$, the response $w_{i}$ is computed as $w_{i}=k_{i}-b_{i}^{\prime} e_{I D^{*}}$ using $A^{\prime}$ 's secret key $e_{I D^{*}}$ for challenge $b_{i}^{\prime}$, that is now computed from $A^{\prime}$ 's challenge $b_{i}^{*}$ through $b_{i}^{\prime \prime}=c_{i} / a_{i}, b_{i}^{\prime}=b_{i}^{*} / b_{i}^{\prime \prime}$.

Claim 2 The output of $\mathbf{E x p}_{1}$ is perfectly indistinguishable from the output of $\mathbf{E x p}_{0}$.

Proof. The only difference between $\mathbf{E x p}_{0}$ and $\mathbf{E x p}_{1}$ is in the order of generation of challenges $b_{i}^{\prime}$ and $b_{i}^{\prime \prime}$ : Generate random $b_{i}^{\prime}$ and set $b_{i}^{\prime \prime}=b^{*} / b_{i}^{\prime}$, or generate random $b_{i}^{\prime \prime}=c_{i} / a_{i}$ and set $b_{i}^{\prime}=b^{*} / b_{i}^{\prime \prime}$. This difference does not affect the resulting distribution of left party's messages and the output of $\mathbf{E x p}_{1}$ distributes exactly as the output of $\mathbf{E x p}_{0}$.

$\mathbf{E x p}_{2}$. Third experiment $\mathbf{E x p}_{2}$ proceeds just as $\mathbf{E x p}_{1}$, except that $\left(c_{i}\right)$ is now selected so that $\left(g^{c_{i}}\right)$ becomes a linear tuple, instead of a random tuple.

In order to prove indistinguishability between the outputs of $\mathbf{E x p}_{2}$ and $\mathbf{E x p}_{1}$, first we prepare a following lemma.

Lemma 1. Under the same assumption as Proposition 3, any feasible adversary A outputs a linear tuple $\left(g_{1}^{*}, \ldots, g_{6}^{*}\right)$ as the one-time commitment key for the right interaction in either of the two execution of the subscheme only with negligible probability in $\mathbf{E x p}_{1}$ (or $\mathbf{E x p}_{2}$ ).

Proof. The proof is similar to the proof of Proposition 2, where we have shown that any feasible sender $\mathcal{S}^{*}$ in a stand-alone setting can generate a linear tuple $\left(g_{1}, \ldots, g_{6}\right)$ as the one-time commitment key only with negligible probability, by exhibiting an invertor $I$ that computes the discrete-log of $g_{c}$ over $g$ with help of the KEA-extractors $H_{c_{i}}$ and $H_{s_{i}}$ using such $\mathcal{S}^{*}$. 
To prove this lemma, we again construct an invertor $I$ that computes the discrete-log of $g_{c}$ over $g$ using such $A$. In addition to the work done by $I$ in the proof of Proposition 2, the new $I$ needs to simulate the honest left party. Since also this $I$, that sets up public keys for simulated $A$, knows $A$ 's secret key $e_{I D^{*}}$, there is no difficulty for this $I$ to simulate the left party in $\mathbf{E x p}_{1}\left(\right.$ or $\left.\mathbf{E x p}_{2}\right)$.

One subtle point is in the construction of new KEA-adversary $H_{c_{i}}$. Recall that the work of $H_{c_{i}}$ is to compute $\left(g_{i}\left(=g^{c_{i}}\right), g_{x}^{c_{i}}\right)$ on input $\left(g, g_{x}\right)$ (and $w_{x}=\left(\delta, g_{c}, d_{x}, e_{y}, b_{1}, \ldots, b_{6}\right.$, coins1)). In the proof of Proposition 2, the trapdoor $d_{x}$ (in $w_{x}$ ), that is generated to satisfy $H(\operatorname{tag})+d_{x} \neq 0$, is used to compute the $g_{x}^{c_{i}}$ by $H_{c_{i}}$. $H_{c_{i}}$ picked up $h_{i}\left(=G_{x}^{c_{i}}\right)$ from the $\mathcal{S}^{*}$ 's second message and computed $g_{x}^{c_{i}}=h_{i}^{1 /\left(H(t a g)+d_{x}\right)}$. (Recall $G_{x}=g_{x}{ }^{H(t a g)+d_{x}}$.) However in the case of this lemma, $H_{c_{i}}$ must deal with the right interaction and need to compute $g_{x}^{c_{i}^{*}}$ from $h_{i}\left(=G_{x}^{* c_{i}^{*}}\right)$ in the right interaction with $G_{x}^{*}=g_{x}^{H\left(\operatorname{tag}^{*}\right)} h_{x}$. Now $t a g^{*}$, that also belongs to the right interaction, is selected by $A$ after it sees CRS (that includes $g, g_{x}$ ). So, we have no guarantee of being $H\left(\operatorname{tag}^{*}\right)+d_{x} \neq 0$ and new $H_{c_{i}}$ cannot mimic the old $H_{c_{i}}$ just doing as $g_{x}^{c_{i}^{*}}=h_{i}^{1 /\left(H\left(t a g^{*}\right)+d_{x}\right)}$.

To remedy this situation, the new $I$ generates $g_{x}$ and $h_{x}$ in CRS in a following manner. First, $I$ selects a random $e_{x}, \eta$ from $\mathbb{Z}_{q}^{*}$, and sets $g_{x}=g^{e_{x}}, h_{x}=g_{x}^{-H(t a g)} g^{\eta}$. (Note $G_{x}=g^{\eta}$ and remember the left tag tag is selected by $A$ before it sees CRS.) Then, $H_{c_{i}}$ can compute $g_{x}^{c_{i}^{*}}$ as $\left(\frac{h_{i}^{*}}{g_{i}^{* \eta}}\right)^{\frac{1}{H(\operatorname{tag})^{*}-H(\operatorname{tag})}}$, using $\eta$ that will be newly added in the auxiliary input $w_{x}$. (Note when $\left(g, G_{x}^{*}, g_{i}^{*}, h_{i}^{*}\right)$ is a DHtuple and $g_{i}^{*}=g^{c_{i}^{*}}$, we have $h_{i}^{*}=\left(G_{x}^{*}\right)^{c_{i}^{*}}=\left(g^{\eta} g_{x}^{H\left(t a g^{*}\right)-H(t a g)}\right)^{c_{i}^{*}}=g_{i}^{* \eta}\left(g_{x}^{c_{i}^{*}}\right)^{H\left(\operatorname{tag}^{*}\right)-H(\operatorname{tag})}$. So, $\left(\frac{h_{i}^{*}}{g_{i}^{* \eta}}\right)^{\frac{1}{H\left(\operatorname{tag}^{*}\right)-H(\operatorname{tag})}}=g_{x}^{c_{i}^{*}}$.) Because $A$ must choose distinct $t a g^{*}$ from tag (to gain nontrivial advantage), it is guaranteed that $H\left(\operatorname{tag}^{*}\right)-H(\operatorname{tag}) \neq 0$.

Claim 3 Under the same assumption as Proposition 3, the output of $\mathbf{E x p}_{2}$ is computationally indistinguishable from the output of $\mathbf{E x p}_{1}$.

The proof is in the same line to the proof of Proposition 1, where in order to show the hiding property a distinguisher between linear and random tuples was constructed and it simulated an honest sender for adversarial receiver $\mathcal{R}^{*}$ to gain its advantage. In the proof of this claim, a new distinguisher does the same simulation for the left interaction for adversary $A$, and in addition it simulates an honest right party and extracts the value committed to by $A$ for the right party in some efficient way, using some KEA-extractors, that is needed for the assumed distinguisher to distinguish between the two experiments.

Proof. Suppose that the output of $\mathbf{E x p}_{2}$ is distinguishable from the output of $\mathbf{E x p}_{1}$ by some efficient distinguisher $D_{12}$ with some non-negligible advantage (with respect to $m$ and $z$ ).

Using such distinguisher $D_{12}$, we construct an efficient (non-uniform) distinguisher $D_{L I N}$ between a pair of linear tuples and a pair of random tuples with non-negligible advantage, that would contradict to the decisional linear assumption on $G$.

Given a pair of tuples $\left(g_{1}, \ldots, g_{6}\right),\left(g_{1}^{+}, \ldots, g_{6}^{+}\right)$as inputs, $D_{L I N}$ simulates the left party for $A$ in such a way that the $\left(g_{1}, \ldots, g_{6}\right),\left(g_{1}^{+}, \ldots, g_{6}^{+}\right)$would occupy one-time commitment keys of the left interactions in the two parallel executions. Note that, due to the modification introduced in $\mathbf{E x p}_{1}$, one-time commitment keys included in the second sender-message are being chosen independently from the first sender-message $\left(A_{i}\right),\left(U_{i}\right)$. So, such simulation is quite easy. After generating the view of $A, D_{L I N}$ invokes KEA-extractors $H_{c_{i}}^{*}$, that are essentially same as the ones used by $I$ in the proof of Lemma 1, to extract coins $c_{i}^{*}$ satisfying $g_{i}^{*}=g^{c_{i}^{*}}$ for the one-time commitment key $\left(g_{i}^{*}\right)$ in 
the right interaction. Because by Lemma 1 the commitment keys $\left(g_{1}^{*}, \ldots, g_{6}^{*}\right)$ can be supposed to be non-linear, $D_{L I N}$ is able to compute $g^{m^{*}}$ (with the determining value $m^{*}$ of $A$ 's commitment in the right interactions) as $g^{m^{*}}=\left(M_{1}^{* 1 / c_{4}^{*}} M_{2}^{* 1 / c_{5}^{*}} M_{3}^{*-1 / c_{6}^{*}}\right)^{1 /\left(c_{1}^{*} / c_{4}^{*}+c_{2}^{*} / c_{5}^{*}-c_{3}^{*} / c_{6}^{*}\right)}$ using those $c_{i}^{*}$, and as well as $g^{+m^{*}}$. This means that $D_{L I N}$ can extract $m^{*}$ by using another KEA-extractor (that corresponds to another KEA-adversary that outputs $\left(g^{m^{*}}, g^{+^{m^{*}}}\right)$ on input $\left.\left(g, g^{+}\right)\right)$and then can invoke the assumed distinguisher $D_{12}$ on $m^{*}$ (and other easy-to-collect items) to distinguish the given tuples. A formal description of $D_{L I N}$ is given in Figure 7.

- Distinguisher $D_{L I N}$ on input $\left(\left(g_{1}, \ldots, g_{6}\right),\left(g_{1}^{+}, \ldots, g_{6}^{+}\right) ;(z, m)\right)$ :

1. (Simulate the left and right parties for $A$.) $D_{L I N}$ invokes $A$ on $z$. Receiving $I D^{*}, I D$ and $\operatorname{tag}$ from $A$, $D_{L I N}$ simulates the left and right parties for $A$ as follows, for each of the two parallel executions of the subscheme.

(a) (Emulate a public/private-key pair and CRS.) $D_{L I N}$ selects random $e_{I D^{*}}$ from $\mathbb{Z}_{q}{ }^{*}$, a random $g$ from $G$ and sets $g_{I D^{*}}=g^{e} e_{D^{*}} . D_{L I N}$ also selects a random $g_{I D}$ from $G$. $D_{L I N}$ gives to $A\left(g_{I D^{*}}, e_{I D^{*}}\right)$ as $A$ 's public/private-key pair and $g_{I D}$ as the right-party's public key. Further, $D_{L I N}$ selects random $e_{c}, e_{x}, e_{y}, \eta$ from $\mathbb{Z}_{q}{ }^{*}$ and sets $g_{c}=g^{e_{c}}, g_{x}=g^{e_{x}}, g_{y}=g^{e_{y}}, h_{x}=g_{x}^{-H(t a g)} g^{\eta}$. (Note that $G_{x}=g^{\eta}$.) $D_{L I N}$ gives $\left(g, g_{c}, g_{x}, h_{x}, g_{y}\right)$ as CRS to $A$.

(b) (Emulate a first left-party message.) Receiving a request from $A, D_{L I N}$ chooses random $\left(s_{i}\right),\left(k_{i}\right),\left(b_{i}^{\prime \prime}\right)$ from $\mathbb{Z}_{q}^{*}$ and computes, for $i=1, \ldots, 6, A_{i}=g_{i}{ }^{1 / b_{i}^{\prime \prime}} g_{c}^{s_{i}}, U_{i}=g^{k_{i}}$. (Note when $g_{i}=g^{c_{i}}$ and $c_{i}=a_{i} b_{i}^{\prime \prime}$, we have $g_{i}{ }^{1 / b_{i}^{\prime \prime}}=g^{a_{i}}$ as in $\operatorname{Exp}_{1}\left(\right.$ or $\left.\operatorname{Exp}_{2}\right)$.) $D_{L I N}$ sends $\left(A_{i}\right),\left(U_{i}\right)$ to $A$.

(c) (Emulate a first right-party message.) Receiving a first message $\operatorname{tag}^{*},\left(A_{i}^{*}\right),\left(U_{i}^{*}\right)$ to the right party from $A, D_{L I N}$ chooses random $\left(b_{i}\right)$ from $\mathbb{Z}_{q}{ }^{*}$ and sends them to $A$.

(d) (Emulate a second left-party message.) Receiving a first message $\left(b_{i}^{*}\right)$ to the left party from $A$, $D_{L I N}$ computes, for $i=1, \ldots, 6, h_{i}=g_{i}{ }^{\eta}, P_{i}=g^{s_{i}}, Q_{i}=g_{y}{ }^{s_{i}}$. (Note when $g_{i}=g^{c_{i}}$, we have $h_{i}=g_{i}^{\eta}=\left(g^{\eta}\right)^{c_{i}}=G_{x}^{c_{i}}$ as in $\operatorname{Exp}_{1}\left(\right.$ or $\left.\mathbf{E x p}_{2}\right)$.) In addition, $D_{L I N}$ computes $w_{i}=k_{i}-b_{i}^{\prime} e_{I D^{*}}$ for $b_{i}^{\prime}=b_{i}^{*} / b_{i}^{\prime \prime}$ for $i=1, \ldots, 6$, and computes homomorphic commitment $M_{1}, M_{2}, M_{3}$ to $m$ using its input $\left(g_{i}\right)$ as commitment key. $D_{L I N}$ sends the second sender-message $\left(g_{i}\right),\left(h_{i}\right), \ldots,\left(M_{i}\right)$ to $A$.

(e) (Complete commitment phases.) Receiving a second message $\left(g_{i}^{*}\right),\left(h_{i}^{*}\right), \ldots,\left(M_{i}^{*}\right)$ to the right party from $A, D_{L I N}$ checks the verifier equation as specified by $\Sigma^{\prime}$. If any of them is not true, $D_{L I N}$ aborts with a random bit. Else let $w=\left(\left(g_{1}, \ldots, g_{6}\right)\right.$, coins $)$, where coins denotes the coins used to simulate this execution of subscheme so far by $D_{L I N}$, except the coins used for generating the first element $g$ in the CRS.

2. (Generate output.) Let $g$ and $g^{+}$be the first elements of CRSs in the two executions of the subscheme. $D_{L I N}$ invokes KEA-extractor $H^{*}$ on $\left(g, g^{+} ; z, w, w^{+}\right)$to get $m^{*}$. Then, $D_{L I N}$ invokes the assumed distinguisher $D_{12}$ on $\left(\left\{\tau, \tau^{+}\right\}\right.$, view $\left.A, m^{*},\left\{(m, r, t),\left(m, r^{+}, t^{+}\right)\right\}\right)$and outputs its output.

Fig. 7. Distinguisher $D_{L I N}$.

As easily seen (from comments in the description), $D_{L I N}$ perfectly simulates the view of $A$ in $\mathbf{E x p}_{1}$ if the input $\left(g_{i}\right),\left(g_{i}^{+}\right)$are random, and perfectly simulates the view of $A$ in $\mathbf{E x p}_{2}$ if the input $\left(g_{i}\right),\left(g_{i}^{+}\right)$are linear. Then, $D_{L I N}$ extracts the value $m^{*}$, that is supposed to be committed to by $A$ for the right party, by using KEA-extractor $H^{*}$. Assuming the extracted $m^{*}$ is in fact the one committed to by $A, D_{L I N}$ is now able to use the assumed distinguisher $D_{12}$ on $m^{*}$ and the others, to distinguish the input tuples are linear or random, contradicting to the assumption.

Thus, now all we need to show is that the KEA-extractor $H^{*}$, that corresponds to following KEA-adversary $H$, extracts the right determining value $m^{*}$ of $A$ 's commitment in the right interaction only with negligible exceptions.

KEA-Adversary $H$ on input $\left(g, g^{+} ; z, m, w, w^{+}\right)$: 
1. (Reproduce the view of $A$.) $H$ invokes $A$ on $z$. Receiving $I D^{*}, I D, \operatorname{tag}$ from $A, H$ reproduces the view of $A$ using the coins given in $w, w^{+}$, for each of the two parallel executions of the subscheme.

2. (Extract coins generated by $A$.) For each of the above two parallel executions, $H$ extracts the coins $\left(c_{i}^{*}\right)$ that defines the one-time commitment key $\left(g_{i}^{*}\right)$ in the right interactions. Namely, $H$ sets $w_{x}=\left(z, e_{I D^{*}}, g_{I D}, g_{c}, \eta, e_{y}, g_{1}, \ldots, g_{6}, \operatorname{coin}_{L}, b_{1}, \ldots, b_{6}, w^{+}\right)$(where notation is the same as in $D_{L I N}$ besides $\operatorname{coin}_{L}$, that denotes the coins used to simulate the left party in this execution) and invokes KEA-extractor $H_{c_{i}}^{*}$ on input $\left(g, g_{x} ; w_{x}\right)$ to obtain $c_{i}^{*}$ satisfying $g_{i}^{*}=g^{c_{i}^{*}}$ for $i=1, \ldots, 6$. Then, $H$ computes $u=\left(M_{1}^{* 1 / c_{4}^{*}} M_{2}^{* 1 / c_{5}^{*}} M_{3}^{*-1 / c_{6}^{*}}\right)^{1 /\left(c_{1}^{*} / c_{4}^{*}+c_{2}^{*} / c_{5}^{*}-c_{3}^{*} / c_{6}^{*}\right)}$. (Note it should be $u=g^{m^{*}}$ with determining value $m^{*}$ of $A$ 's homomorphic commitment in this execution.)

3. (Generate output.) Output a pair of extracted values $\left(u, u^{+}\right)$of the two executions.

Given $\left(g, g^{+}\right)$as input, KEA-adversary $H$ uses the coins given in the auxiliary input $w, w^{+}$to reproduce the exact view of $A$ in $D_{L I N}$ and then extracts coins $c_{i}^{*}$ generated by $A$, that are expected to satisfy $g_{i}^{*}=g^{c_{i}^{*}}$ by using KEA-extractors $H_{c_{i}}^{*}$ on $\left(g, g_{x}\right)$ and

$$
w_{x}=\left(z, e_{I D^{*}}, g_{I D}, g_{c}, \eta, e_{y}, g_{1}, \ldots, g_{6}, \operatorname{coin}_{L}, b_{1}, \ldots, b_{6}, w^{+}\right) .
$$

For a while, assume the coins $c_{i}^{*}$ are right. Then, since $\left(g_{1}^{*}=g^{c_{1}^{*}}, \ldots, g_{6}^{*}=g^{c_{6}^{*}}\right)$ is not linear by Lemma 1 (only with negligible exceptions), it defines perfectly binding homomorphic commitment and so $H$ can compute the value $u=\left(M_{1}^{* 1 / c_{4}^{*}} M_{2}^{* 1 / c_{5}^{*}} M_{3}^{*-1 / c_{6}^{*}}\right)^{1 /\left(c_{1}^{*} / c_{4}^{*}+c_{2}^{*} / c_{5}^{*}-c_{3}^{*} / c_{6}^{*}\right)}$ that must be equal to $g^{m^{*}}$ with $m^{*}=\operatorname{det}\left(M_{1}^{*}, M_{2}^{*}, M_{3}^{*}\right)$. The output of $H$ is a pair $\left(u, u^{+}\right)$of such $u$ for the two executions of the subscheme. Thus, $H$ outputs $\left(g^{m^{*}}, g^{m^{m^{*}}}\right)$ on input $\left(g, g^{+}\right)$, and since clearly $\log _{g}\left(g^{+}\right)$is independent from the auxiliary input $\left(z, m, w, w^{+}\right)$, we see the corresponding $H^{*}$ extracts the right determining value $m^{*}$ of $A$ 's commitment only with negligible exceptions, by the knowledge of exponent assumption, as desired.

Now, all we have to do is to show the above KEA-extractors $H_{c_{i}}^{*}$, that correspond to the following KEA-adversaries, extract the right coins $c_{i}^{*}$ satisfying $g_{i}^{*}=g^{c_{i}^{*}}$ only with negligible exceptions. As in the proof of Lemma 1 , given $\left(g, g_{x}\right)$ as input, $H_{c_{i}}$ reproduces the exact view of $A$ invoked in $H$ using the auxiliary input $w_{x}$, and picks up $g_{i}^{*}$ and $h_{i}^{*}$ from the view and outputs $\left(g_{i}^{*}\left(=g^{c_{i}^{*}}\right),\left(\frac{h_{i}^{*}}{g_{i}^{* \eta}}\right)^{\frac{1}{H\left(\operatorname{tag}^{*}\right)-H(t a g)}}\left(=g_{x}^{c_{i}^{*}}\right)\right)$. Namely, $H_{c_{i}}$ outputs $\left(g^{c_{i}^{*}}, g_{x}^{c_{i}^{*}}\right)$ on input $\left(g, g_{x}\right)$. In addition, as directly verified, all items in $w_{x}$ are independent of the discrete-log $e_{x}$ of $g_{x}$ over $g$. Hence, by the knowledge of exponent assumption, $H_{c_{i}}^{*}$ in $H$ must output $c_{i}^{*}$ satisfying $g_{i}^{*}=g^{c_{i}^{*}}$ only with negligible exception. This completes the proof of Claim 3.

$\mathbf{E x p}_{3}$. In the final experiment $\mathbf{E x p}_{3}$, the simulated left party is changed to commit to a dummy value $m_{0}$ through homomorphic commitment $M_{1}=g_{1}^{m_{0}} g_{4}^{r_{0}}, M_{2}=g_{2}^{m_{0}} g_{5}^{t_{0}}, M_{3}=g_{3}^{m_{0}} g_{6}^{r_{0}+t_{0}}$, instead of committing to the true value $m$, for each of the two executions of subscheme. Since by the modification introduced in $\mathbf{E x p}_{2}$ the one-time commitment key $\left(g_{i}\left(=g^{c_{i}}\right)\right)$ of the left interaction is linear, its decommitment to the true value $m$ (to be included in the output) exists and is computed by $r=r_{0}+\frac{c_{1}}{c_{4}}\left(m_{0}-m\right), t=t_{0}+\frac{c_{2}}{c_{5}}\left(m_{0}-m\right)$. In addition, in $\mathbf{E x p}_{3}$, the computation of the determining value $m^{*}=\operatorname{det}\left(M_{1}^{*}, M_{2}^{*}, M_{3}^{*}\right)$ of $A$ 's commitment is changed. It is now efficiently extracted by using KEA-extractor $H^{*}$ on $\left(g, g^{+} ; z, m, w, w^{+}\right)$with $w=\left(e_{I D^{*}}, g_{I D}, \tau,\left(A_{i}\right),\left(U_{i}\right),\left(b_{i}\right),\left(g_{i}\right),\left(b_{i}^{\prime}\right),\left(M_{i}\right)\right)$.

Claim 4 The output of $\mathbf{E x p}_{3}$ is statistically indistinguishable from the output of $\mathbf{E x p}_{2}$. 
Proof. In $\mathbf{E x p}_{2}$, the homomorphic commitment $M_{1}, M_{2}, M_{3}$ in the left interaction is perfectly hiding and equivocal since the used one-time commitment key $g_{1}, \ldots, g_{6}$ is linear. So, it is trivial to see the view ${ }_{A}$ in $\mathbf{E x p}_{3}$ is exactly distributed as the view $w_{A}$ in $\mathbf{E x p}_{2}$.

Then, all we need to show is that given input $\left(g, g^{+} ; z, m, w, w^{+}\right), H^{*}$ in $\mathbf{E x p}_{3}$ correctly outputs the determining value $m^{*}=\operatorname{det}\left(M_{1}^{*}, M_{2}^{*}, M_{3}^{*}\right)$ of $A^{\prime}$ 's commitment in the right interaction only with negligible exceptions. This $H^{*}$ is similar as $H^{*}$ used by $D_{L I N}$ in the proof of Claim 3. However, there is an important difference. As seen below, the new $H$ (that corresponds to the new $H^{*}$ ) also uses extractors $H_{c_{i}}^{*}$ of $c_{i}^{*}$ on input $\left(g, g_{x}\right)$. However, in the current setting, the information given to this $H_{c_{i}}^{*}$ through auxiliary input $w_{x}$ will contain (part of) $A$ 's view $\left(A_{i}\right),\left(U_{i}\right),\left(b_{i}\right),\left(g_{i}\right),\left(b_{i}^{\prime}\right),\left(M_{i}\right)$, instead of the used coins as before. So, as to the new $H_{c_{i}}$, there is no direct guarantee that the discrete-log $e_{x}$ of the given input $\left(g_{x}, g\right)$ is independent of the given auxiliary input $w_{x}$. (The left party's second message, especially $\left(b_{i}^{\prime}=b_{i}^{*} / b_{i}^{\prime \prime}\right)$, depends on the $A$ 's challenge $\left(b_{i}^{*}\right)$, that possibly depends on the CRS, that includes $g, g_{x}$. ) So, before describing the new KEA-adversary $H$, we need to give an equivalent code for simulating the left party in $\mathbf{E x p}_{3}$, to avoid such problem.

Fix arbitrary left party's first message $\left(A_{i}\right),\left(U_{i}\right)$ and arbitrary right party's first message $\left(b_{i}\right)$ in $\mathbf{E x p}_{3}$. (Note then $A$ 's challenge $\left(b_{i}^{*}\right)$ for the left party is also fixed.) Then, in $\mathbf{E x p}_{3}$, the second left-party message $\left(g_{i}\right),\left(h_{i}\right), \ldots,\left(w_{i}\right)$ (excluding $\left(M_{i}\right)$ which is clearly independent of $\left.g, g_{x}\right)$ is being generated by the following procedure. (Note the fixed $\left(U_{i}=g^{k_{i}}\right)$ implicitly determines $k_{i}$.)

- The original procedure:

1. Take a random tuple $\left(c_{1}, \ldots, c_{6}\right)$ so that $\left(g_{1}=g^{c_{1}}, \ldots, g_{6}=g^{c_{6}}\right)$ is linear and a random tuple $\left(s_{1}, \ldots, s_{6}\right)$ from $\mathbb{Z}_{q}^{*}$. (Note $s_{i}$ implicitly determines $a_{i}$ through the fixed $A_{i}=g^{a_{i}} g_{c}^{s_{i}}$.)

2. For $i=1$ to 6 , compute $g_{i}=g^{c_{i}}, h_{i}=G_{x}^{c_{i}}, P_{i}=g^{s_{i}}, Q_{i}=g_{y}^{s_{i}}, b_{i}^{\prime \prime}=c_{i} / a_{i}, b_{i}^{\prime}=b_{i}^{*} / b_{i}^{\prime \prime}, w_{i}=$ $k_{i}-b_{i}^{\prime} e_{I D^{*}}$.

This procedure is re-written as follows, using trapdoor information $\tau$ and the zero-knowledge simulator of proof $P_{i}$.

- The second procedure:

1. Take a linear tuple $\left(g_{1}, \ldots, g_{6}\right)$ from $G$ and a random tuple $\left(b_{1}^{\prime \prime}, \ldots, b_{6}^{\prime \prime}\right)$ from $\mathbb{Z}_{q}$.

2. For $i=1$ to 6 , compute $h_{i}=g_{i}^{\eta}, P_{i}=\left(A_{i} g_{i}^{-1 / b_{i}^{\prime \prime}}\right)^{1 / e_{c}}, Q_{i}=P_{i}^{e_{y}}, b_{i}^{\prime}=b_{i}^{*} / b_{i}^{\prime \prime}, w_{i}=k_{i}-b_{i}^{\prime} e_{I D^{*}}$, with $\eta=e_{x} \cdot\left(H(\operatorname{tag})+d_{x}\right)$.

We can select random $\left(b_{i}^{\prime}\right)$, instead of random $\left(b_{i}^{\prime \prime}\right)$, preserving the distribution and the above procedure is re-written as follows.

- The third procedure:

1. Take a linear tuple $\left(g_{1}, \ldots, g_{6}\right)$ from $G$ and a random tuple $\left(b_{1}^{\prime}, \ldots, b_{6}^{\prime}\right)$ from $\mathbb{Z}_{q}$.

2. For $i=1$ to 6 , compute $h_{i}=g_{i}^{\eta}, b_{i}^{\prime \prime}=b_{i}^{*} / b_{i}^{\prime}, P_{i}=\left(A_{i} g_{i}^{-1 / b_{i}^{\prime \prime}}\right)^{1 / e_{c}}, Q_{i}=P_{i}^{e_{y}}, w_{i}=k_{i}-b_{i}^{\prime} e_{I D^{*}}$.

The third procedure shows that the second left-party message includes the three independent random components $\left(g_{i}\right),\left(b_{i}^{\prime}\right),\left(M_{i}\right)$ and the other items $\left(h_{i}\right),\left(P_{i}\right),\left(Q_{i}\right),\left(w_{i}\right)$ are determined by them. Thus, we see that the discrete-log $e_{x}$ of $g_{x}$ over $g$ is independent of the part of view $A$ consisting of $\left(A_{i}\right),\left(U_{i}\right),\left(b_{i}\right)$ and $\left(g_{i}\right),\left(b_{i}^{\prime}\right),\left(M_{i}\right)$.

Now we describe the KEA-adversary $H$ that uses the above third procedure to simulate the left-party messages.

KEA-Adversary $H$ on input $\left(g, g^{+} ; z, w=\left(e_{I D^{*}}, g_{I D}, \tau,\left(A_{i}\right),\left(U_{i}\right),\left(b_{i}\right),\left(g_{i}\right),\left(b_{i}^{\prime}\right),\left(M_{i}\right)\right), w^{+}\right)$: 
1. (Reproduce the view of $A$.) $H$ invokes $A$ on $z$. Receiving $I D^{*}, I D, \operatorname{tag}$ from $A, H$ reproduces the view of $A$ using $w$ as follows, for each of the two parallel executions of the subscheme.

(a) (Emulate a public/private-key pair and CRS.) $H$ takes trapdoor information $\tau$ from $w$ to regenerate $A$ 's public/private-key pair $\left(g_{I D^{*}}, e_{I D^{*}}\right)$, right-party's public key $g_{I D}$ and CRS $\left(g, g_{c}, g_{x}, h_{x}, g_{y}\right)$, and gives them to $A$. $H$ records $\eta=e_{x} \cdot\left(H(t a g)+d_{x}\right)$ for later use.

(b) (Emulate a first left-party message.) Receiving a request for a first left-party message from $A, H$ takes $\left(A_{i}\right),\left(U_{i}\right)$ from $w$ and sends them to $A$.

(c) (Emulate a first right-party message.) Receiving a first message $\operatorname{tag}^{*},\left(A_{i}^{*}\right),\left(U_{i}^{*}\right)$ to the rightparty from $A, H$ takes $\left(b_{i}\right)$ from $w$ and sends them to $A$.

(d) (Emulate a second left-party message.) Receiving a first message $\left(b_{i}^{*}\right)$ to the left-party from $A, H$ takes $\left(g_{i}\right),\left(b_{i}^{\prime}\right),\left(M_{i}\right)$ from $w$, computes $h_{i}=g_{i}^{\eta}, b_{i}^{\prime \prime}=b_{i}^{*} / b_{i}^{\prime}, P_{i}=\left(A_{i} g_{i}^{-1 / b_{i}^{\prime \prime}}\right)^{1 / e_{c}}, Q_{i}=$ $P_{i}^{e_{y}}, w_{i}=k_{i}-b_{i}^{\prime} e_{I D^{*}}$, and sends $\left(g_{i}\right),\left(h_{i}\right),\left(P_{i}\right),\left(Q_{i}\right),\left(b_{i}^{\prime}\right),\left(w_{i}\right),\left(M_{i}\right)$ to $A$.

(e) (Extract coins generated by $A$.) Receiving the second message $\left(g_{i}^{*}\right),\left(h_{i}^{*}\right), \ldots,\left(M_{i}^{*}\right)$ to the right-party from $A, H$ extracts the coins $\left(c_{i}^{*}\right)$ that $A$ generated. Namely, $H$ sets $w_{x}=$ $\left(z, e_{I D^{*}}, g_{I D}, e_{c}, \eta, e_{y},\left(A_{i}\right),\left(U_{i}\right),\left(b_{i}\right),\left(g_{i}\right),\left(b_{i}^{\prime}\right),\left(M_{i}\right), v i e w_{A}^{+}\right)$(where view $w_{A}^{+}$denotes the view of $A$ in the another execution of the subscheme) and invokes KEA-extractor $H_{c_{i}}^{*}$ on input $\left(g, g_{x} ; w_{x}\right)$ to obtain $c_{i}^{*}$, for $i=1, \ldots, 6$.

(f) (Extract $g^{m^{*}}$ generated by $A$.) $H$ computes $u=\left(M_{1}^{* 1 / c_{4}^{*}} M_{2}^{* 1 / c_{5}^{*}} M_{3}^{*-1 / c_{6}^{*}}\right)^{1 /\left(c_{1}^{*} / c_{4}^{*}+c_{2}^{*} / c_{5}^{*}-c_{3}^{*} / c_{6}^{*}\right)}$. 2. (Generate output.) Return a pair of the extracted values $\left(u, u^{+}\right)$at Step $1 \mathrm{f}$.

Given $\left(g, g^{+}\right)$as input, KEA-adversary $H$ reproduces the exact view of $A$ in $\mathbf{E x p}_{3}$ by using the third procedure on those independent random components given in the auxiliary input $w$, and then extracts $A^{\prime}$ 's coins $c_{i}^{*}$ that are expected to satisfy $g_{i}^{*}=g^{c_{i}^{*}}$ by KEA-extractor $H_{c_{i}}^{*}$ on $\left(g, g_{x}\right)$ and $w_{x}=\left(z, e_{I D^{*}}, g_{I D}, e_{c}, \eta, e_{y},\left(A_{i}\right),\left(U_{i}\right),\left(b_{i}\right),\left(g_{i}\right),\left(b_{i}^{\prime}\right),\left(M_{i}\right)\right.$, view $\left.w_{A}^{+}\right)$. For a while, assume the expectations are right. Then, since $\left(g_{1}^{*}=g^{c_{1}^{*}}, \ldots, g_{6}^{*}=g^{c_{6}^{*}}\right)$ is not linear by Lemma 1, it defines perfectly binding homomorphic commitment and so $H$ can compute the value $u=$ $\left(M_{1}^{* 1 / c_{4}^{*}} M_{2}^{* 1 / c_{5}^{*}} M_{3}^{*-1 / c_{6}^{*}}\right)^{1 /\left(c_{1}^{*} / c_{4}^{*}+c_{2}^{*} / c_{5}^{*}-c_{3}^{*} / c_{6}^{*}\right)}$ that must be equal to $g^{m^{*}}$ with $m^{*}=\operatorname{det}\left(M_{1}^{*}, M_{2}^{*}, M_{3}^{*}\right)$. The output of $H$ is a pair $\left(u, u^{+}\right)$of such $u$ for the two executions of the subscheme. Thus, $H$ outputs $\left(g^{m^{*}}, g^{m^{*}}\right)$ on $\left(g, g^{+}\right)$. Since clearly the discrete-log of $g^{+}$over $g$ is independent from the auxiliary input $\left(z, m, w, w^{+}\right)$, by the knowledge of exponent assumption, the corresponding KEA-extractor $H^{*}$ extracts the right determining value $m^{*}$ of $A$ 's commitment only with negligible exceptions, as desired.

Now, all we have to do is to show that the KEA-extractor $H_{c_{i}}^{*}$, that corresponds to the following KEA-adversary $H_{c_{i}}$, extracts the coins $c_{i}^{*}$ satisfying $g_{i}^{*}=g^{c_{i}^{*}}$ only with negligible exceptions. Given $\left(g, g_{x}\right)$ as input, $H_{c_{i}}$ reproduces the exact view of $A$ invoked in $H$ using the auxiliary input $w_{x}$ by using the third procedure, and picks up $g_{i}^{*}$ and $h_{i}^{*}$ from them and outputs $\left(g_{i}^{*}\left(=g^{c_{i}^{*}}\right),\left(h_{i}^{*} / g_{i}^{* \eta}\right)^{1 /\left(H\left(\operatorname{tag}^{*}\right)-H(\operatorname{tag})\right)}\left(=g_{x}^{c_{i}^{*}}\right)\right)$. As discussed before, the items in $w_{x}$ (especially the ones that belongs to the view of $A$ ) was chosen to be independent of the discrete $\log e_{x}$ of $g_{x}$ with $g$. Hence, by the knowledge of exponent assumption, $H_{c_{i}}^{*}$ outputs $c_{i}^{*}$ satisfying $g_{i}^{*}=g^{c_{i}^{*}}$ only with negligible exceptions. That completes the proof of Claim 4.

By Claims 2, 3 and 4, Equation (6) is satisfied by EQV and EXT in Figure 8, that are immediate from the description of $\mathbf{E x p}_{3}$ and the third procedure, completing the proof of Proposition 3 . 
- Simulator EQV on input $\left(\operatorname{tag}, e_{I D^{*}}, m_{0}\right)$ does as follows, for each of the two parallel executions of the subscheme:

1. (Emulate a first left-party message.) Choose random $a_{i}, s_{i}, k_{i}$ from $\mathbb{Z}_{q}^{*}$ and compute $A_{i}=g^{a_{i}} g_{c}^{s_{i}}, U_{i}=g^{k_{i}}$ for $i=1, \ldots, 6$. Send $A_{1}, \ldots, A_{6}, U_{1}, \ldots, U_{6}$.

2. (Emulate a second left-party message.) Receiving a first message $\left(b_{i}^{*}\right)$, select random $c_{i}$ from $\mathbb{Z}_{q}^{*}$ so that $\left(g^{c_{i}}\right)$ becomes a linear tuple and compute $g_{i}=g^{c_{i}}, h_{i}=G_{x}{ }^{c_{i}}, P_{i}=g^{s_{i}}, Q_{i}=g_{y}{ }^{s_{i}}$ for $i=1, \ldots, 6$ with $G_{x}=g_{x}{ }^{H(\operatorname{tag})} h_{x}$. In addition, compute $b_{i}^{\prime \prime}=c_{i} / a_{i}, b_{i}^{\prime}=b_{i}^{*} / b_{i}^{\prime \prime}, w_{i}=k_{i}-b_{i}^{\prime} e_{I D^{*}}$ for $i=1, \ldots, 6$. Then, take random $r_{0}, t_{0}$ from $\mathbb{Z}_{q}$ and compute $M_{1}=g_{1}^{m_{0}} g_{4}^{r_{0}}, M_{2}=g_{2}^{m_{0}} g_{5}^{t_{0}}, M_{3}=g_{3}^{m_{0}} g_{6}^{r_{0}+t_{0}}$. Send $\left(g_{i}\right),\left(h_{i}\right),\left(P_{i}\right),\left(Q_{i}\right),\left(b_{i}^{\prime}\right),\left(w_{i}\right),\left(M_{i}\right)$.

3. (Output an honest decommitment.) Receiving a true value $m$, compute $r=r_{0}+\frac{c_{1}}{c_{4}}\left(m_{0}-m\right), t=$ $t_{0}+\frac{c_{2}}{c_{5}}\left(m_{0}-m\right)$, and output $m, r, t$.

- Extractor EXT on input $\left(\tau, \tau^{+}\right.$, view $\left.A\right)$ does as follows:

1. For each of the two parallel executions of the subscheme, take necessary items from view $A$ and set $w=\left(e_{I D^{*}}, g_{I D}, \tau,\left(A_{i}\right),\left(U_{i}\right),\left(b_{i}\right),\left(g_{i}\right),\left(b_{i}^{\prime}\right),\left(M_{i}\right)\right)$. (Note the secret key $e_{I D^{*}}$ is in view $\left.w_{A}\right)$

2. Invoke the KEA-extractor $H^{*}$ (in the proof of Claim 4) on $\left(g, g^{+} ; z, w, w^{+}\right)$to get and output $m^{*}$.

Fig. 8. EQV and EXT. 


\section{B A full description of $\operatorname{Exp}_{0}$}

Experiment $\operatorname{Exp}_{0}$ on input $m \in \mathbb{Z}_{q}$ and $z \in\{0,1\}^{*}:$

1. (Simulate left and right parties for $A$.) Invoke $A$ on $z$. Receiving $I D^{*}, I D$ and $\operatorname{tag}$ from $A$, simulate the left and right parties for $A$ as follows, for each of the two parallel executions of the subscheme.

(a) (Emulate a public/private-key pair and CRS for $A$.) Select a random $e_{I D^{*}}$ from $\mathbb{Z}_{q}$ and a random element $g$ from $G$, and set $g_{I D^{*}}=g^{e_{I D^{*}}}$. Select a random element $g_{I D}$ from $G$. Give to $A\left(g_{I D^{*}}, e_{I D^{*}}\right)$ as $A$ 's pair of public/private key and $g_{I D}$ as the right-party's public key. In addition, choose $e_{c}, e_{x}, d_{x}, e_{y}$ randomly from $\mathbb{Z}_{q}$, set $g_{c}=g^{e_{c}}, g_{x}=g^{e_{x}}, h_{x}=g_{x}^{d_{x}}, g_{y}=g^{e_{y}}$, and give $\left(g, g_{c}, g_{x}, h_{x}, g_{y}\right)$ as CRS to $A$.

(b) (Emulate a first left-party message.) Receiving a request for a first left-party message from $A$, choose $a_{1}, \ldots, a_{6}, s_{1}, \ldots, s_{6}, w_{1}, \ldots, w_{6}, b_{1}^{\prime}, \ldots, b_{6}^{\prime}$ randomly from $\mathbb{Z}_{q}^{*}$ and compute $A_{i}=g^{a_{i}} g_{c}^{s_{i}}, U_{i}=g^{w_{i}} g_{I D^{*}} b_{i}^{\prime}$ for $i=1, \ldots, 6$. Send $A_{1}, \ldots, A_{6}, U_{1}, \ldots, U_{6}$ to $A$.

(c) (Emulate a first right-party message.) Receiving a first message $\operatorname{tag}^{*}, A_{1}^{*}, \ldots, A_{6}^{*}, U_{1}^{*}, \ldots, U_{6}^{*}$ to the rightparty from $A$, choose $b_{1}, \ldots, b_{6}$ randomly from $\mathbb{Z}_{q}{ }^{*}$ and send them to $A$.

(d) (Emulate a second left-party message.) Receiving a first message $b_{1}^{*}, \ldots, b_{6}^{*}$ to the left-party from $A$, compute $b_{i}^{\prime \prime}=b_{i}^{*} / b_{i}^{\prime}, c_{i}=a_{i} b_{i}^{\prime \prime}, g_{i}=g^{c_{i}}, h_{i}=G_{x}{ }^{c_{i}}, P_{i}=g^{s_{i}}, Q_{i}=g_{y}{ }^{s_{i}}$ for $i=1, \ldots, 6$ with $G_{x}=$ $g_{x}{ }^{H(t a g)} h_{x}$. Then, take two random $r, t$ from $\mathbb{Z}_{q}$ and compute $M_{1}=g_{1}^{m} g_{4}^{r}, M_{2}=g_{2}^{m} g_{5}^{t}, M_{3}=g_{3}^{m} g_{6}^{r+t}$. Send $\left(g_{i}\right),\left(h_{i}\right),\left(P_{i}\right),\left(Q_{i}\right),\left(b_{i}^{\prime}\right),\left(w_{i}\right),\left(M_{i}\right)$ to $A$.

(e) (Complete commitment phases.) Receiving a second message $\left(g_{i}^{*}\right),\left(h_{i}^{*}\right),\left(P_{i}^{*}\right),\left(Q_{i}^{*}\right),\left(b_{i}^{* \prime}\right),\left(w_{i}^{*}\right),\left(M_{i}^{*}\right)$ to the right-party from $A$, compute $b_{i}^{\prime \prime}=b_{i} / b_{i}^{\prime *}$ and check

$$
\operatorname{DH}\left(g, G_{x}^{*}, g_{i}^{*}, h_{i}^{*}\right), \operatorname{DH}\left(g, g_{c}, P_{i}^{*}, Q_{i}^{*}\right), U_{i}^{*}=g^{w_{i}^{*}} g_{I D}{ }^{b_{i}^{*}}, e\left(A_{i}^{*}, g^{b^{\prime \prime *}}\right)=e\left(g_{i}^{*}, g_{c}\right) e\left(P_{i}^{*}, g_{c}^{b^{\prime \prime *}}\right)
$$

with $G_{x}^{*}=g_{x}^{H\left(\operatorname{tag}^{*}\right)} h_{x}$ for $i=1, \ldots, 6$. If any of them (for any $i$ ) is not true, abort with output $\perp$.

2. (Generate output.) Let view $_{A}$ be the view of $A$ after completing the above commitment phase. If the two values of $m^{*}=\operatorname{det}\left(M_{1}^{*}, M_{2}^{*}, M_{3}^{*}\right)$ determined in the two parallel executions are the same, return $\left(\left(\tau, \tau^{+}\right)\right.$, view $\left._{A}, m^{*},\left((m, r, t),\left(m, r^{+}, t^{+}\right)\right)\right)$, otherwise return $\perp$. 